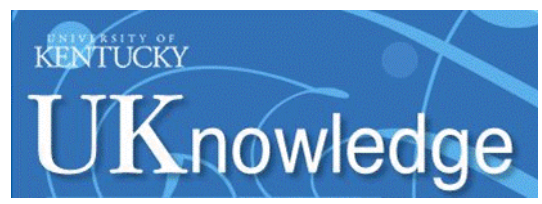

University of Kentucky

UKnowledge

\title{
Spectroscopic Characterization of Galaxy Clusters in RCS-1: Spectroscopic Confirmation, Redshift Accuracy, and Dynamical Mass-Richness Relation
}

\author{
David G. Gilbank \\ North-West University, South Africa \\ L. Felipe Barrientos \\ Pontificia Universidad Católica de Chile, Chile \\ Erica Ellingson \\ University of Colorado Boulder \\ Kris Blindert \\ University of Toronto, Canada \\ H. K. C. Lee \\ University of Toronto, Canada \\ Follow this and additional works at: https://uknowledge.uky.edu/physastron_facpub \\ See rart of the the fortropdysicicsal andh Astronomy Commons \\ Right click to open a feedback form in a new tab to let us know how this document benefits you.
}

\section{Repository Citation}

Gilbank, David G.; Barrientos, L. Felipe; Ellingson, Erica; Blindert, Kris; Lee, H. K. C.; Anguita, T.; Gladders, M. D.; Hall, P. B.; Hertling, G.; Infante, L.; Yan, Renbin; Carrasco, M.; Garcia-Vergara, Cristina; Dawson, K. S.; Lidman, C.; and Morokuma, T., "Spectroscopic Characterization of Galaxy Clusters in RCS-1:

Spectroscopic Confirmation, Redshift Accuracy, and Dynamical Mass-Richness Relation" (2018). Physics and Astronomy Faculty Publications. 562.

https://uknowledge.uky.edu/physastron_facpub/562

This Article is brought to you for free and open access by the Physics and Astronomy at UKnowledge. It has been accepted for inclusion in Physics and Astronomy Faculty Publications by an authorized administrator of UKnowledge. For more information, please contact UKnowledge@lsv.uky.edu. 
Spectroscopic Characterization of Galaxy Clusters in RCS-1: Spectroscopic Confirmation, Redshift Accuracy, and Dynamical Mass-Richness Relation

Digital Object Identifier (DOI)

https://doi.org/10.1093/mnras/sty355

Notes/Citation Information

Published in Monthly Notices of the Royal Astronomical Society, v. 476, issue 2, p. 1991-2012.

This article has been accepted for publication in Monthly Notices of the Royal Astronomical Society (๑): 2018 The Author(s). Published by Oxford University Press on behalf of the Royal Astronomical Society. All rights reserved.

The copyright holders have granted the permission for posting the article here.

Authors

David G. Gilbank, L. Felipe Barrientos, Erica Ellingson, Kris Blindert, H. K. C. Lee, T. Anguita, M. D. Gladders, P. B. Hall, G. Hertling, L. Infante, Renbin Yan, M. Carrasco, Cristina Garcia-Vergara, K. S. Dawson, C. Lidman, and T. Morokuma 


\title{
Spectroscopic characterization of galaxy clusters in RCS-1: spectroscopic confirmation, redshift accuracy, and dynamical mass-richness relation
}

\author{
David G. Gilbank, ${ }^{1 \star}$ L. Felipe Barrientos, ${ }^{2}$ Erica Ellingson, ${ }^{3}$ Kris Blindert, ${ }^{4}$ \\ H. K. C. Yee, ${ }^{4}$ T. Anguita, ${ }^{5}$ M. D. Gladders, ${ }^{6}$ P. B. Hall, ${ }^{7}$ G. Hertling, ${ }^{2}$ L. Infante, ${ }^{2}$ \\ R. Yan, ${ }^{8}$ M. Carrasco, ${ }^{2,9}$ Cristina Garcia-Vergara, ${ }^{2,10}$ K. S. Dawson, ${ }^{11}$ C. Lidman ${ }^{12}$ \\ and T. Morokuma ${ }^{13}$
}

Affiliations are listed at the end of the paper

Accepted 2017 December 6. Received 2017 October 27; in original form 2017 May 30

\begin{abstract}
We present follow-up spectroscopic observations of galaxy clusters from the first Red-sequence Cluster Survey (RCS-1). This work focuses on two samples, a lower redshift sample of $\sim 30$ clusters ranging in redshift from $z \sim 0.2-0.6$ observed with multiobject spectroscopy (MOS) on 4-6.5-m class telescopes and a $z \sim 1$ sample of $\sim 10$ clusters 8 -m class telescope observations. We examine the detection efficiency and redshift accuracy of the now widely used redsequence technique for selecting clusters via overdensities of red-sequence galaxies. Using both these data and extended samples including previously published RCS-1 spectroscopy and spectroscopic redshifts from SDSS, we find that the red-sequence redshift using simple twofilter cluster photometric redshifts is accurate to $\sigma_{z} \approx 0.035(1+z)$ in RCS- 1 . This accuracy can potentially be improved with better survey photometric calibration. For the lower redshift sample, $\sim 5$ per cent of clusters show some (minor) contamination from secondary systems with the same red-sequence intruding into the measurement aperture of the original cluster. At $z \sim 1$, the rate rises to $\sim 20$ per cent. Approximately ten per cent of projections are expected to be serious, where the two components contribute significant numbers of their red-sequence galaxies to another cluster. Finally, we present a preliminary study of the mass-richness calibration using velocity dispersions to probe the dynamical masses of the clusters. We find a relation broadly consistent with that seen in the local universe from the WINGS sample at $z \sim 0.05$.
\end{abstract}

Key words: galaxies: clusters: general-galaxies: distances and redshifts.

\section{INTRODUCTION}

The importance of galaxy clusters as both test-beds of galaxy evolution and probes of cosmology is well established (e.g. Eke, Cole \& Frenk 1996; Borgani \& Guzzo 2001; Majumdar \& Mohr 2004). In recent years, wide-field surveys have started producing huge numbers of galaxy clusters (e.g. Koester et al. 2007; Rykoff et al. 2014), in greater numbers than all those previously discovered, and thus it is of great importance to understand the sample selection for these experiments and the accuracy and limitations of the various survey methods.

The traditional approach to galaxy cluster discovery has been optical searches for overdensities of galaxies (Abell 1958). In recent years, colour-based variations (e.g. Gal et al. 2000; Gladders
\& Yee 2000; Miller et al. 2005) of Abell's original method have led to decreased false positive rates from unrelated line-of-sight projections of clusters, and high completenesses. This has led to a resurgence of optical/NIR searches for these 'galaxy selected' clusters, especially given the relatively high efficiency of only needing to obtain multicolour (or even single colour) imaging data. Indeed, several on-going and future large surveys are planned to build large cluster samples using such techniques, e.g. the Dark Energy Survey (DES ${ }^{1}$ - Flaugher 2005), the Panoramic Survey Telescope and Rapid Response Systems (Pan-STARRS ${ }^{2}$ - Chambers et al. 2016), and the Large Synoptic Survey Telescope (LSST ${ }^{3}$ - LSST Science Collaboration et al. 2009).

\footnotetext{
${ }^{1}$ http://www.darkenergysurvey.org

${ }^{2} \mathrm{http}: / /$ pan-starrs.ifa.hawaii.edu

${ }^{3} \mathrm{http} / / / \mathrm{www} .1$ sst.org
} 
At the same time, X-ray based surveys, which search for the signature of the hot intra-cluster medium (ICM) of galaxy clusters, have also made great leaps forward. Samples are now beginning to span hundreds of square degrees at moderate to high redshifts, $z \gtrsim$ 0.5 .

Aside from the vast increase in depth and area compared with previous surveys, there has been an expansion in the wavelength domain explored by large surveys, particularly extending down to $\mathrm{mm}$ and sub-mm wavelengths. This, in turn, has led to the possibility of using new techniques, which probe different physical properties of the clusters, in order to select them. For example, there are a number of large experiments (Hasselfield et al. 2013; Planck Collaboration XXIX 2013; Bleem et al. 2015) exploiting the thermal Sunyaev-Zeldovich (SZ, Sunyaev \& Zeldovich 1972) effect. This idea has long been prized as a method for selecting galaxy clusters as it depends linearly on the gas density of the ICM (compared with X-ray luminosity selection that depends on gas density ${ }^{2}$ and temperature ${ }^{1 / 2}$ ) and should essentially mass-select clusters independently of their redshift. It is useful to group together X-ray luminosity-selected and SZ effect-selected clusters, as both these 'gas selected' cluster surveys detect clusters in a way that is fundamentally different from the 'galaxy selected' samples described above. However, it is important to note that the majority of such surveys still rely on optical/NIR imaging follow-up to look for overdensities of galaxies in order to confirm the nature of the source and to estimate its redshift. ${ }^{4}$

Once a cluster sample has been built, for both galaxy evolution and studies of cosmology (as well as studies of cluster physics), it is of interest to be able to derive properties such as the masses of the clusters. In many cases, there is a trade-off between obtaining relatively accurate mass estimates at the expense of detailed follow-up studies of a small sub-sample of clusters and using an observationally cheaper mass proxy, which may be derived for all clusters in the sample, but at the expense of much higher uncertainties on each individual mass estimate. For example, for X-ray studies, the preferred mass estimator would be a quantity like $Y_{\mathrm{X}}$ (Kravtsov, Vikhlinin \& Nagai 2006), which ideally requires sufficient $\mathrm{S} / \mathrm{N}$ in the $\mathrm{X}$-ray spectrum to be able to determine an accurate $\mathrm{X}$-ray temperature, $T_{\mathrm{X}}$, along with sufficient $\mathrm{S} / \mathrm{N}$ and resolution in the imaging to be able to excise any regions that may bias this temperature estimate (e.g. cool cores), and to determine the gas density from the surface brightness profile. Similarly, the gas fraction $\left(f_{\text {gas }}\right)$ is often used when sufficient counts are available or, in the presence of lower counts but still good spatial resolution, excising the core region from a simple luminosity estimate, $L_{\mathrm{X}}$, leads to a relatively low scatter mass proxy (see, e.g. Pratt et al. 2009, and references therein). In the absence of this, the next resort would be a more approximate estimate of $T_{\mathrm{X}}$ without these refinements. Finally, in the absence of sufficient $\mathrm{S} / \mathrm{N}$ for this, simply an X-ray luminosity measurement, $L_{\mathrm{X}}$, with some assumed scaling between mass and luminosity is typically used. The latter can be determined from any survey data sufficient to find the cluster in the first place (assuming an estimate of the redshift is available from some other source, such as optical spectroscopy, of course). Similarly for galaxy-selected surveys, high quality mass measurements require observationally expensive follow-up from, for example, optical spectroscopy and/or deep, high-resolution imaging for weak lensing (although, for low

\footnotetext{
${ }^{4}$ The exception being where the X-ray spectrum is able to yield a redshift from Fe lines, such as the K- or L-shell complexes (e.g. Yu et al. 2011 and references therein)
}

redshift or relatively massive clusters, weak lensing masses may be obtained from the survey data themselves, e.g. Kettula et al. 2015). In the absence of these, a mass proxy from the survey data must be used, such as optical richness (counts of numbers of galaxies per cluster).

The cross-comparison of galaxy-selected and gas-selected cluster samples on a largescale for the first time is slowly leading to the realization that the physical properties of samples selected with different techniques are strongly influenced by the selection method (Donahue et al. 2001; Gilbank et al. 2004; Hicks et al. 2013; Rozo et al. 2014; Saro et al. 2015). Using all-sky data from the Planck satellite and its overlap with the galaxy-selected cluster survey, maxBCG (Koester et al. 2007), Planck Collaboration XII (2011) examined the SZ signal-to-optical richness scaling relations $\left(Y_{500}-N_{200}\right)$. They found that their observed relation has a lower normalization than would be predicted from $L_{X}$-selected samples. Explanations to reconcile this discrepancy between the gas-galaxy properties of galaxy-selected and gas-selected samples have been proposed by Rozo et al. (2014), Angulo et al. (2012), and Sehgal et al. (2013), among others, the essence of which is the importance of modelling self-consistently the constraints on the scaling relations between the joint distributions in optical richness, $L_{\mathrm{X}}$, etc. and cluster mass (see also Maughan 2014). We will explore this subject in future papers, and this forms the motivation framing the presentation of our results for the galaxy-selected cluster survey, the first Red-sequence Cluster Survey (RCS-1) in this paper.

Hence, survey properties such as the false positive rate, redshift accuracy, and mass proxy accuracy are crucial to understand for all science derived from cluster surveys. The red-sequence technique (Gladders \& Yee 2000, and variations thereof) has become one of the de facto standards of galaxy-selected cluster surveys. Here, we present the results of a large, multiyear campaign aimed at characterizing RCS-1. These results focus on two spectroscopic follow-up samples: one for $z \lesssim 0.5$ and one for $z \sim 1$ candidate clusters.

The layout for this paper is as follows. In Section 2, we describe the follow-up cluster sample selection (including updates to the cluster catalogues) and the follow-up spectroscopic data; in Section 3 , we show the comparison between the RCS-1 cluster catalogues and the confirmation spectroscopy; Section 4 examines the redshift accuracy of the red-sequence technique; Section 5 measures velocity dispersions as mass estimates for a subsample of clusters with sufficient member galaxies and discusses several important effects related to the red-sequence selection of cluster and their potential impact on ultimate derivation of the mass-richness relation. The main results are summarized in Section 6.

All magnitudes are quoted on the $\mathrm{AB}$ system unless otherwise stated, and we assume a cosmology $\left(h, \Omega_{\mathrm{M}}, \Omega_{\lambda}\right)=(0.7,0.3,0.7)$.

\section{OBSERVATIONS AND DATA REDUCTION}

RCS-1 (Gladders \& Yee 2005) is a $70 \mathrm{deg}^{2}$ imaging survey using the mosaic imagers CFH12K on CFHT and MOSAIC on the CTIO 4-m, in $R$ and $z$, designed to find galaxy clusters out to $z \sim 1$. As mentioned above, it is a key to understand survey properties such as the false positive rate, redshift accuracy, and mass proxy accuracy (i.e. the mass-richness relation).

\subsection{Sample selection}

Cluster candidates were selected for spectroscopic follow-up early on in the RCS project, before final cluster catalogues were available. 


\subsubsection{Updates to the RCS cluster catalogue}

The RCS-1 photometric catalogues were recalibrated in 2011 March. This featured a number of improvements over the catalogues used in previous work and implemented a more accurate colour and magnitude calibration. This improved photometric calibration used the colour of the stellar locus and 2MASS photometry in a manner similar to that described in Gilbank et al. (2011) for RCS-2 (but, since RCS-1 contains only two filters, $R$ and $z^{\prime 5}$ ) this recalibration used 2MASS colours to better isolate the stellar locus. By comparison with overlapping SDSS photometry, the improved catalogues are accurate to $\approx 0.027 \mathrm{mag}$ in colour and $\approx 0.057 \mathrm{mag}$ in magnitude $\left(z^{\prime}\right){ }^{6}$

The revision of the photometric catalogues also means that the properties of the cluster catalogues are modified. The redshift estimate is based on the $\left(R-z^{\prime}\right)$ colour and the richness, in turn, depends on the number of red galaxies down to some magnitude limit. In order to simply recover the updated properties of the clusters, a 'stripped down' version of the red-sequence cluster finding was run following the approach described in Lu et al. (2009). Briefly, the magnitude-based weighting and the radial profile weighting of the method described in Gladders \& Yee (2000) are removed, and so the detection of galaxy clusters is simply a case of counting overdensities of galaxies whose colour errors are compatible with being members of a model red-sequence. The overdensity is simply a count of the number of galaxies within a $0.5 \mathrm{Mpc}$ physical radius top-hat filter at the model redshift, and the errors are calculated from Poisson statistics in the counts of cluster+background galaxies and the measured field-to-field variation. The significance of a detection, $\sigma_{\mathrm{RCS}}$, is calculated directly from the overdensity and errors (see $\mathrm{Lu}$ et al. 2009; Barrientos et al., in preparation, for more details). For the nominal cluster catalogue, a cut-off significance of $\sigma_{\mathrm{RCS}} \geq 5.0$ is used, but the significance maps are calculated down to $3.0 \sigma$, and it will be necessary to explore some of these lower levels in parts of this work. Finally, the cluster centre is refined by searching for the luminosity weighted centre around the (richness selected) nominal position. For the purposes of verification, in this paper, the centre used is that of the visually identified BCG. BCGs were selected by looking for the brightest galaxy that appeared close to an overdensity of bright, candidate red-sequence galaxies. In most cases, this was unambiguous. Where two bright galaxies appeared equally good candidates, the average of their positions was used, and in a small number of cases, no BCG could be identified, in which case the centre of the overdensity of bright galaxies was used. The exact choice of centre only affects the measurement of richness, and then only if the radius used to measure richness is comparable with the size of the centring uncertainty. Richness is calculated by counting the number of galaxies compatible with the red-sequence colour slice down to $M^{\star}+1$ in a $1 \mathrm{Mpc}$ radius circle. For the $1 \mathrm{Mpc}$ radius we adopt here, the choice of centre has negligible impact on the measured richness. For this work, this richness measurement is chosen for its simplicity. Clusters for which the centring choice might make a difference, for example, merging/disturbed or projected systems, are flagged and discussed as special cases in Section 3.

Finally, it is important to note that in this stripped-down version of the cluster-finding algorithm, the photometric redshift range

\footnotetext{
${ }^{5}$ Although note that the RCS-1 $z$-band imaging is deeper than that of RCS-2.

${ }^{6}$ This may sound counter-intuitive, but the colour has a smaller statistical uncertainty since it is a higher $\mathrm{S} / \mathrm{N}$ measurement (it uses a smaller aperture) than the total magnitude, and the total magnitude calibration is tied to the colour calibration (see Gilbank et al. 2011 for details).
}

covered is firmly restricted to $0.20<z_{\mathrm{RCS}}<0.985$, as these are the limits at which the $(R-z)$ colours start to become degenerate. Previous versions of the RCS- 1 catalogue attempted to extend this range, which in part appeared to be successful due to the additional luminosity weighting applied.

Throughout this paper, the naming convention used in the previous generation of the RCS- 1 cluster catalogues is used, so that already published clusters ${ }^{7}$ may be readily identified herein, but updated positions, photometric redshifts, and richnesses are presented.

\subsection{CFHT and Magellan spectroscopy}

For the lower redshift $\left(0.2 \lesssim z \lesssim 0.6^{8}\right)$ sample, observations were carried out using two instruments. In the north, we used the multi-object spectroscopy (MOS) arm of the Multi-Object Spectrograph/Subarcsecond Imaging Spectrograph (Le Fevre et al. 1994) operating on the 3.6-m Canada-France-Hawaii telescope; hereafter, this instrumental set-up is referred to as the CFH-MOS. It provides a $9 \times 8 \operatorname{arcmin}^{2}$ field of view for spectroscopy, and we chose a grism with moderate dispersion, which yielded a spectral resolution of $13.8 \AA$ for slits of width 1.35 arcsec. We also employed a bandlimiting filter to allow for multitiering of spectra on the detector, which greatly improves spatial sampling as well as the total number of objects that can be observed in one mask. Our redshift range is approximately the same as that of the $\mathrm{CNOC} 2$ field galaxy redshift survey (Yee et al. 2000), which was performed on the same instrument; we therefore used the CNOC2 band-limiting filter (for a transmission curve, see fig. 2 of Yee et al. 2000). This filter shortens spectra to only $2000 \AA$, allowing us to place over 100 slits on each MOS mask, a factor of $\sim 3$ improvement over observing without the band-limiting filter.

In the south, we used the Low Dispersion Survey Spectrograph 2 (Allington-Smith et al. 1994) on the 6.5-m Magellan telescopes (hereafter referred to as Mgl-LDSS2). This instrument has a spectroscopic field of view of $6 \times 4 \operatorname{arcmin}^{2}$, and grisms at slightly lower dispersion than CFH-MOS for a spectral resolution of $15.4 \AA$ for slits of width 1.25 arcsec. Two medium-dispersion grisms were available for this instrument, one with more red sensitivity than the other; we used the blue grism for clusters at redshifts $z<0.4$, and switched to the red grism for higher redshift clusters. Unfortunately, no band-limiting filter was available for Mgl-LDSS2, so we were limited to an average of 33 slits per mask. The observational strategy followed Yee, Ellingson \& Carlberg (1996) with typically $1 \mathrm{~h}$ observations, and total integration times ranging from $1 \mathrm{~h}$ for the bright masks at $z \sim 0.2$ to $4 \mathrm{~h}$ for the fainter masks at $z \sim 0.6$.

\subsection{VLT spectroscopy}

At the VLT, FORS2 was used in MXU MOS mode in order to place a large number of slits and also the freedom to have them with different lengths and inclinations. We used the 300I grism in conjunction with the OG590 filter giving a dispersion of $1.62 \AA \mathrm{pixel}^{-1}$ and a wavelength coverage from 6500 to $9500 \AA$. A slit width of 0.8 arcsec and a slit length of 8 arcsec were used for most objects. Masks were created using the FORS instrument mask simulation program. Galaxies were selected mostly using a fixed magnitude limit; however, IR colour information was used when available.

\footnotetext{
${ }^{7}$ In particular, the $z \sim 1$ clusters form a large fraction of the HST Supernova Cosmology Project (Dawson et al. 2009) sample.

${ }^{8}$ With one cluster at $z=0.66$.
} 
Table 1. Properties of zlo follow-up sample. Columns are: short cluster name, preliminary RCS-1 name, mean cluster spectroscopic redshift, central RA, Dec., physical offset between centre of spectroscopic overdensity and RCS-1 catalogue position, $z_{\mathrm{RCS}}$, RCS cluster catalogue significance, short cluster name.

\begin{tabular}{|c|c|c|c|c|c|c|c|}
\hline Short name & Cluster name & $\mathrm{Z}_{\text {spec }}$ & $\mathrm{RA}\left({ }^{\circ}\right)$ & Dec. $\left({ }^{\circ}\right)$ & Offset (Mpc) & $z_{\mathrm{RCS}}$ & $\sigma_{\mathrm{RCS}}$ \\
\hline zlo_001 & J022331 0118.4 & 0.4403 & 35.874035 & 1.308205 & 0.0647 & 0.43 & 9.66 \\
\hline zlo_002 & J022516 0011.5 & 0.3295 & 36.315957 & 0.192051 & 0.1950 & 0.352 & 7.05 \\
\hline zlo_003 & $\mathrm{J} 022402-0227.8$ & 0.3576 & 36.013814 & -2.4607 & 0.0841 & 0.382 & 9.5 \\
\hline zlo_004 & J033414-2824.6 & 0.6642 & 53.560965 & -28.405207 & 0.1217 & 0.63 & 12.81 \\
\hline zlo_005As & J035139-0956.3As & 0.1678 & 57.908204 & -9.940928 & $(0.2867)$ & $(0.289)$ & $(10.73)$ \\
\hline zlo_005B & J035139-0956.3B & 0.3054 & 57.908204 & -9.940928 & 0.2822 & 0.289 & 10.73 \\
\hline zlo_006A(s) & J044207-2815.0As & 0.4108 & 70.617027 & -28.309209 & 0.3338 & 0.409 & 5.88 \\
\hline zlo_006B & J044207-2815.0B & 0.4663 & 70.532099 & -28.256385 & 0.0492 & 0.43 & 13.96 \\
\hline zlo_007 & J051536-4325.5 & 0.4245 & 78.899418 & -43.418142 & 0.0391 & 0.409 & 10.86 \\
\hline zlo_008 & J051919-4247.8 & 0.5744 & 79.842005 & -42.795525 & 0.0427 & 0.538 & 6.82 \\
\hline zlo_009 & J092821 3646.5 & 0.3926 & 142.089519 & 36.780143 & 0.0470 & 0.382 & 12.7 \\
\hline zlo_010s & $\mathrm{J} 0928303646.0 \mathrm{~s}$ & 0.1402 & 142.089519 & 36.780143 & $(0.5746)$ & $(0.382)$ & $(12.7)$ \\
\hline zlo_011 & J093010 3841.2 & 0.4306 & 142.549734 & 38.675612 & 0.1806 & 0.409 & 5.44 \\
\hline zlo_012s & $\mathrm{J} 1120512527.6 \mathrm{~s}$ & 0.3069 & 170.209822 & 25.465524 & 0.289 & 0.5911 & 6.01 \\
\hline zlo_013 & J112038 2522.1 & 0.2621 & 170.169514 & 25.379632 & 0.4341 & 0.250 & 5.69 \\
\hline zlo_015A & J132523 2919.4A & 0.4291 & 201.364683 & 29.33793 & 0.3614 & 0.495 & 10.44 \\
\hline zlo_015Bs & J132523 2919.4Bs & 0.2904 & 201.364683 & 29.33793 & - & 0.382 & 5.382 \\
\hline zlo_016 & J144632 0859.2 & 0.2381 & 221.656823 & 9.033257 & 0.1544 & 0.211 & 6.00 \\
\hline zlo_017 & $\mathrm{J} 1447080949.0$ & 0.2022 & 221.779887 & 9.818905 & 0.1623 & 0.211 & 18.8 \\
\hline zlo_018 & J161547 3057.3 & 0.4186 & 243.95096 & 30.958245 & 0.2777 & 0.382 & 13.08 \\
\hline zlo_019 & J162008 3046.7 & 0.2976 & 245.03633 & 30.79146 & 0.3318 & 0.321 & 7.59 \\
\hline zlo_020 & $\mathrm{J} 211519-6309.5$ & 0.2261 & 318.823277 & -63.151178 & 0.0647 & 0.321 & 6.12 \\
\hline zlo_021s & $\mathrm{J} 211945-6209.8 \mathrm{~s}$ & 0.3595 & 319.923026 & -62.161546 & 0.409 & 0.5722 & 8.05 \\
\hline zlo_022 & $\mathrm{J} 212005-6204.8$ & 0.3258 & 319.958015 & -62.033106 & 0.2879 & 0.430 & 5.52 \\
\hline zlo_023 & $\mathrm{J} 212134-6335.8$ & 0.2171 & 320.417144 & -63.59183 & 0.0425 & 0.211 & 11.04 \\
\hline zlo_024 & $\mathrm{J} 215700-0441.9$ & 0.1668 & 329.247265 & -4.693135 & 0.0624 & 0.289 & 12.11 \\
\hline zlo_025 & $\mathrm{J} 2315440052.8$ & 0.3321 & 348.928896 & 0.886681 & 0.0622 & 0.352 & 16.43 \\
\hline zlo_026 & $\mathrm{J} 231736-0103.0$ & 0.2009 & 349.390571 & -1.056378 & 0.0690 & 0.211 & 9.75 \\
\hline zlo_027 & $\mathrm{J} 231830-0024.5$ & 0.3799 & 349.623305 & -0.408947 & 0.0313 & 0.352 & 9.56 \\
\hline zlo_028 & $\mathrm{J} 234356-3517.5$ & 0.4945 & 355.971349 & -35.28907 & 0.1548 & 0.511 & 10.93 \\
\hline zlo_029 & $\mathrm{J} 234748-3535.1$ & 0.263 & 356.95903 & -35.584098 & 0.0348 & 0.43 & 22.86 \\
\hline
\end{tabular}

We devoted $3 \mathrm{~h}$ per mask, including overheads. The observations were split into three or six individual observations, keeping the total observing time under the nominal $3 \mathrm{~h}$ and to improve cosmic ray rejection. The observations for this program were made during periods $70,72,73$, and 77 .

The spectra were reduced with IRAF in the usual manner. For typical spectra, the wavelength range spans from $\sim 6500$ to $\sim 9000 \AA$ with a dispersion of $3.2 \AA$ pixel $^{-1}$, and an rms uncertainty in the wavelength solution of $0.12 \AA$.

\subsection{Gemini spectroscopy}

GMOS South was used in MOS mode taking advantage of the nod and shuffle capability of the instrument. This allowed the efficient subtraction of the numerous emission sky lines in the red part of the spectrum, where the most common optical spectral features for identification were expected to be found. We used the R150 grating with the $G 5326$ filter, providing a resolution of $\sim 20 \AA$. Slits of $0.75 \operatorname{arcsec}$ width and 2.5 arcsec length were used. Total integrations were about $3 \mathrm{~h}$ per mask, and only one mask per cluster. The observations for this program were made during semesters 2003B, 2004A, and 2004B.

\subsection{Additional spectroscopy}

As part of the HST Cluster Supernova Survey (Dawson et al. 2009), additional MOS was obtained on six clusters using FORS1 and
FORS2 on the VLT, DEIMOS on the Keck II telescope, and FOCAS on Subaru. Details on the observations, the processing of the data, and the determination of the redshifts can be found in Dawson et al. (2009), Morokuma et al. (2010), and Meyers et al. (2012).

\section{CLUSTER IDENTIFICATION}

\subsection{Lower $z$ sample}

For the lower $z$ sample $(0.2 \lesssim z \lesssim 0.6)$, the identification of structures in redshift space and their association with the photometric RCS cluster candidates is relatively straightforward, since this sample possesses relatively large numbers of redshifts for each cluster. The matches to the photometric cluster catalogue and the associated properties are summarized in Table 1, including short-hand names for easy reference, prefixed (zlo_). Several systems were identified in the spectroscopy, which were not the primary target of the follow-up. These serendipitous clusters are designated with the suffix 's' on the cluster name. In some cases, these are projections of significant systems along the line of sight, which may or may not be resolved into separate objects in the photometric cluster catalogue. In the case of such spectroscopic pairs, the two components are designated ' $\mathrm{A}$ ' and ' $\mathrm{B}$ ' in order of increasing redshift. In other cases, these serendipitous clusters are lower significance systems, possibly groups or low richness clusters, which were not identified as cluster candidates by the cluster finding algorithm. 

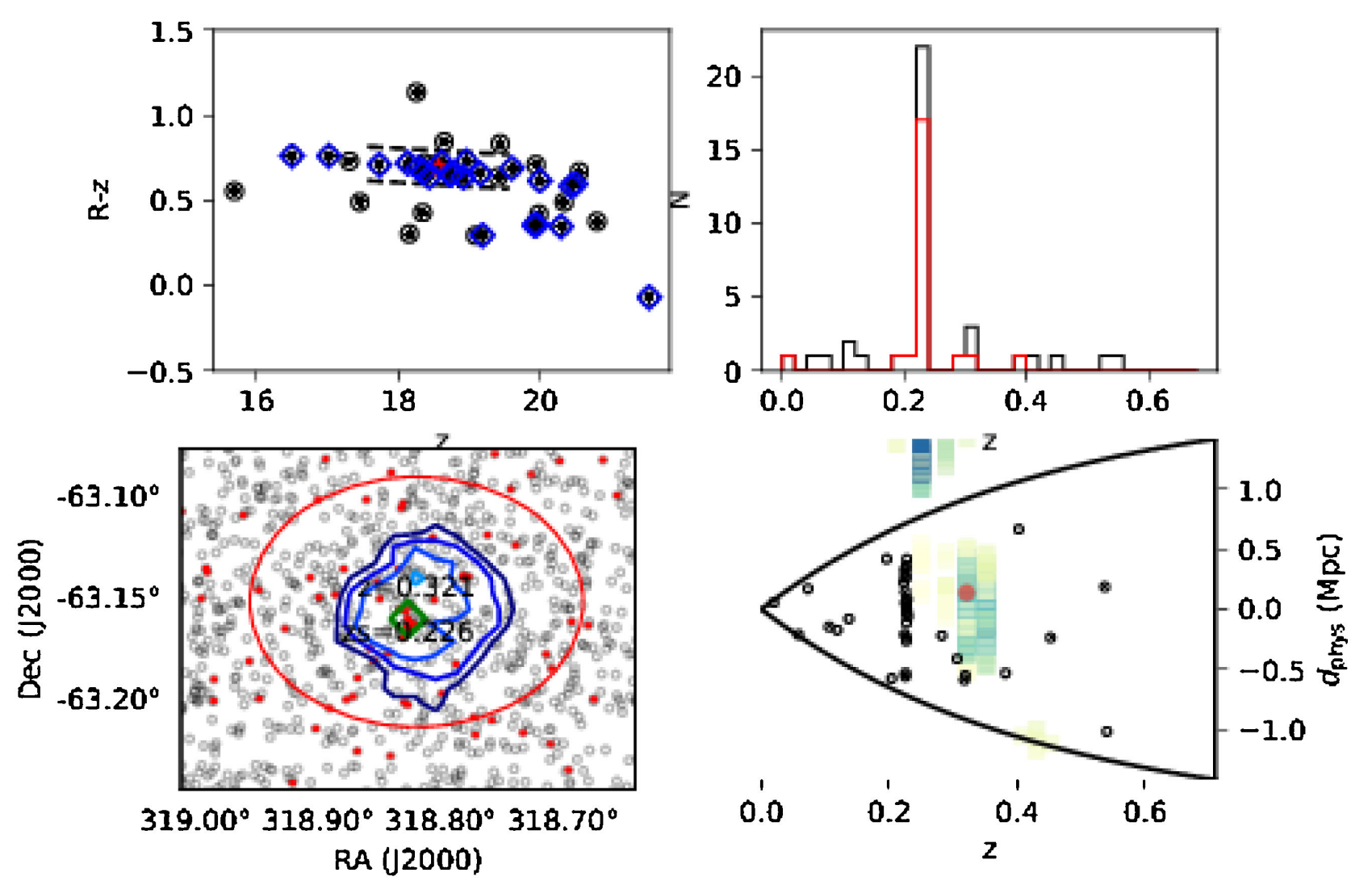

Figure 1. Plots showing CMD (top left-hand panel), redshift histogram (top right-hand panel), RA, Dec. positions (bottom left-hand panel), redshift 'pie diagram in RA slice (bottom right-hand panel) for a typical cluster, J212134-6335.8 (zlo_023). For the CMD, filled black circles denote all galaxies with spectroscopic redshifts; blue diamonds show galaxies within 1 arcmin of the cluster position from the whole photometric catalogue (i.e. including those without spectra); the dashed lines and red star show the position of the best candidate model red-sequence slice and $M^{\star}$, respectively. For the redshift histogram, the black histogram denotes all galaxies; the red histogram is galaxies with colours compatible with the red-sequence slice at the cluster's photometric redshift $P($ on red-sequence $)>0.1$. For the spatial plot, open circles show galaxies in the photometric catalogue and filled red circles denote red-sequence candidate members from the photometry alone; contours denote $\sigma_{\mathrm{RCS}}$ starting at $3 \sigma$ in $1 \sigma$ intervals; the red circle is $1.0 \mathrm{Mpc}$ radius physical at the redshift of the cluster; the redshift labels indicate the photometric redshift $(z)$ and spectroscopic redshift $(z s)$ of systems in the field. On the pie diagram, black points denote all spectroscopic redshifts, the red point denotes the position of the cluster in the photometric cluster catalogue (i.e. $z$ RCS). Shaded squares show significance of each voxel in the cluster finder, starting at $3.0 \sigma$.

In order to verify the association of photometric and spectroscopically detected systems, a way is needed to both visualize the $3 \mathrm{D}$ properties of these two data sets and to associate the individual objects responsible for causing the photometric overdensity with those within the spectroscopic overdensity. i.e. one would like to know the spatial position/extent and location in redshift space of the cluster, traced by the overdensity of galaxies (either red galaxies in the photometric catalogue or spectroscopic redshifts in the spectroscopic catalogue); and the same information for the individual galaxies that make up these candidate overdensities in order to investigate the purity of the technique. These are shown for a typical cluster as the four panels in Fig. 1. The top left-hand panel of the figure shows the colour-magnitude distribution of galaxies within 1 arcmin of the photometric cluster centre with the spectroscopic members (or near-field galaxies) overplotted. Note that since no colour restrictions were placed on the spectroscopic targets, one would not expect only galaxies with the colours of the red-sequence model (indicated) to be found. The top right-hand panel shows a redshift histogram for all spectroscopic data, with the galaxies identified as candidate red-sequence members (i.e. those with colour errors that make them compatible with the colour slice indicated in the previous panel) highlighted. If every red-sequence candidate galaxy was truly a red-sequence cluster member, then a single spike in the redshift histogram would occur at the redshift of the cluster. The two remaining panels show a view of the sky positions of the galaxies in the photometric catalogue, red-sequence candidate cluster members, and photometric significance map contours (left-hand panel), and a redshift-declination 'pie' diagram with spectroscopic redshifts indicated and photometric cluster significance maps overlaid. These can be compared with the positions of the galaxies with redshifts compatible with the spectroscopic overdensity. If the cluster comprised entirely red sequence galaxies, then every galaxy indicated as a spectroscopic member would also be labelled as a candidate red-sequence member. ${ }^{9}$ At these redshifts,

\footnotetext{
${ }^{9}$ It is worth noting that the probability threshold for counting a galaxy as a red-sequence candidate member is low (10 percent). This is done to maximize the chances of including every reasonable candidate member. In calculating final cluster quantities, such as richness, the same cut is applied to the background sample, so the net number of members should always be consistent with any chosen probability threshold (but the uncertainty will formally be larger, the smaller the probability cut). However, when considering only candidate cluster members, as here, this conservative cut likely also includes some foreground and background galaxies. In practise,
} 
fewer than five background galaxies are expected to have colours mimicking red-sequence cluster members within the $1 \mathrm{Mpc}$ counting radius used, relatively independent of redshift over the whole range considered in this paper.

In this example shown in Fig. 1 (zlo_023), it can clearly be seen that a single dominant peak is present in the spectroscopic redshift histogram, and that most of the candidate red-sequence galaxies fall in this peak (top right-hand panel). The galaxies with spectroscopic redshifts show a clear colour-magnitude relation, which also agrees with the red-sequence demonstrated by all (photometrically selected) galaxies in the central arcmin around the cluster position (top right-hand panel). These spectroscopic and red-sequence galaxies show a similar spatial distribution (bottom left-hand panel). And finally, these spatial and redshift concentrations show only a single, dominant overdensity in this survey field (bottom right-hand panel), coincident with the single highest contour level in the photometric cluster significance map.

\subsubsection{Clusters requiring more consideration}

Several systems require more consideration than the cleaner cases above. These include cases comprising secondary clusters projected along the line of sight and lower significance systems that may fall below the formal threshold of the current or previous catalogue.

(i) zlo_005 appears as two systems in the spectroscopic catalogue $\left(z_{\text {spec }}=0.1678\right.$ and 0.3054$)$. A single system is seen in the photometric cluster catalogue and so Table 1 repeats the target cluster's properties in parentheses for the serendipitous component. The higher redshift component is close to the expected redshift of $z_{\mathrm{RCS}}=$ 0.289 . The lower redshift component is below the nominal $z_{\mathrm{RCS}}=$ 0.20 redshift cut-off, however, the redshift histogram in Fig. 2 shows that many of the galaxies in the $z=0.16$ spike have colours compatible with the higher redshift red-sequence. Thus, red-sequence richness and significance estimates could be enhanced by this additional line-of-sight cluster.

(ii) zlo_006 also shows two components $\left(z_{\mathrm{spec}}=0.4108,0.4663\right)$. However, both of these are correctly separated in the new RCS cluster catalogue. The 'A component is marked as '(s) as this was not found in the original RCS algorithm used to select follow-up clusters and so was then serendipitous, but was retrodicted using the current cluster-finding algorithm.

(iii) zlo_009,010s is a similar situation to zlo_005 in that one of the two components (zlo_010s, $z_{\text {spec }}=0.1402$ ) is below the nominal $z_{\mathrm{RCS}}=0.20$ limit. However, in this case, its redshift spike in Fig. 2 does not comprise significant numbers of galaxies with colours of the higher redshift red-sequence. This suggests that the red-sequence measures of zlo_009 are not severely contaminated by the lower redshift component. Thus, it seems reasonable to neglect zlo_010s since it would not be expected to be found in the photometric data, and does not bias the properties of the target cluster.

In summary, all significant overdensities identified in the spectroscopic redshift catalogues were also found by the red-sequence finder with the exception of two systems below the nominal redshift limit (only one of these exhibited galaxies of colours that would bias the red-sequence properties measured for the higher redshift target cluster).

almost all galaxies have a probability of 1.0 or 0.0 , and so the effect of using this 0.10 cut-off is negligible in these plots.

\section{$3.2 z \sim 1$ sample}

The $z \sim 1(0.8 \lesssim z \lesssim 1.1)$ data require more attention to associate the spectroscopic results with the photometric cluster catalogue. In several cases, due to the preliminary nature of the cluster catalogue when the spectroscopic follow-up was undertaken, the clusters are less rich and less significant than those used in the current RCS cluster catalogue. In addition, due to the relative difficulty of obtaining redshifts of $z \sim 1$ galaxies, the spectroscopic statistics are much lower than for the lower redshift sample. In order to examine and validate the candidates, the same plots are examined as for the lower redshift clusters. These are shown in Fig. 3.

For ease of reference, the shorthand, z1_is used to refer to either a spectroscopic overdensity or an RCS cluster candidate or the two combined (when associated). ' $* *$ ' in front of the name indicates that the cluster in not formally identified as a candidate $\left(\sigma_{\mathrm{RCS}} \geq 5.0\right)$ in the revised cluster catalogue. Results are summarized in Table 2.

The following clusters from the $\sigma_{\mathrm{RCS}} \geq 5.0$ photometric cluster catalogue are considered spectroscopically confirmed with no apparent ambiguity/projections: z1_001, z1_004, z1_007, z1_015, and z1_019. Additionally, z1_013 looks like a good match to the only $z \sim 1$ RCS cluster candidate in this field; z1_014 is the label given to the second spike in the spectroscopic redshift histogram, but these redshifts do not appear to be spatially concentrated and so are unlikely to be a missed cluster. Cleanly confirmed clusters that just fell below the $5.0 \sigma_{\mathrm{RCS}}$ catalogue limit are: z1_002, z1_008, and z1_009. The spectroscopic overdensity associated with z1_016 appears as a $\sigma_{\mathrm{RCS}}=5.5$ detection in the photometric cluster significance maps, but no cluster candidate formally appears in the catalogue, likely due to the way in which the significance map was deblended.

\subsubsection{Cases requiring more consideration}

(i) z1_011 is associated with the larger red-sequence overdensity, but z1_012 lies nearer to the centre of the spectroscopic field and was the original target of the spectroscopy. z1_011 has fewer high quality spectra associated with it (three class 1-3 plus another two class 4) due to its location further from the centre of the field. Given the much lower significance in the RCS data cube and its relative lack of spectroscopically confirmed red-sequence galaxies, it appears that $z 1 \_012\left(z_{\text {spec }}=1.073\right)$ is a candidate for a much poorer cluster or group, and so it is unsurprising that it does not appear as a candidate in the updated cluster catalogue. In the updated cluster catalogue, it has been absorbed into the low significance tail of the nearby, slightly lower redshift $\left(z_{\text {spec }}=0.854\right)$ z1_011 system. They are close enough together on the sky that the more minor object (z1_012) will contribute some red-sequence galaxies into a $1 \mathrm{Mpc}$ radius aperture used to measure richness for $\mathrm{z} 1 \_011$, and contaminate red-sequence measurements to some extent. With the minimal spectroscopy available, we cannot accurately estimate the extent of this contamination, but it is likely relatively small.

(ii) z1_017 and z1_018 show two photometric cluster candidates at lower redshifts than the nominal $z \sim 1(z=0.775$ and 0.621 , respectively), there are one or two galaxies at each of these spectroscopic redshifts, plus a handful of galaxies around $z \sim 1$; thus, it is difficult to untangle these systems and not enough to definitively confirm these systems, so they are dropped from further analysis.

(iii) z1_020 shows a single, marginally detected spectroscopic$z$ overdensity but at quite a large redshift offset and significantly spatially offset from the RCS candidate, hinting that this is not the correct association between the spectroscopic and photometric 

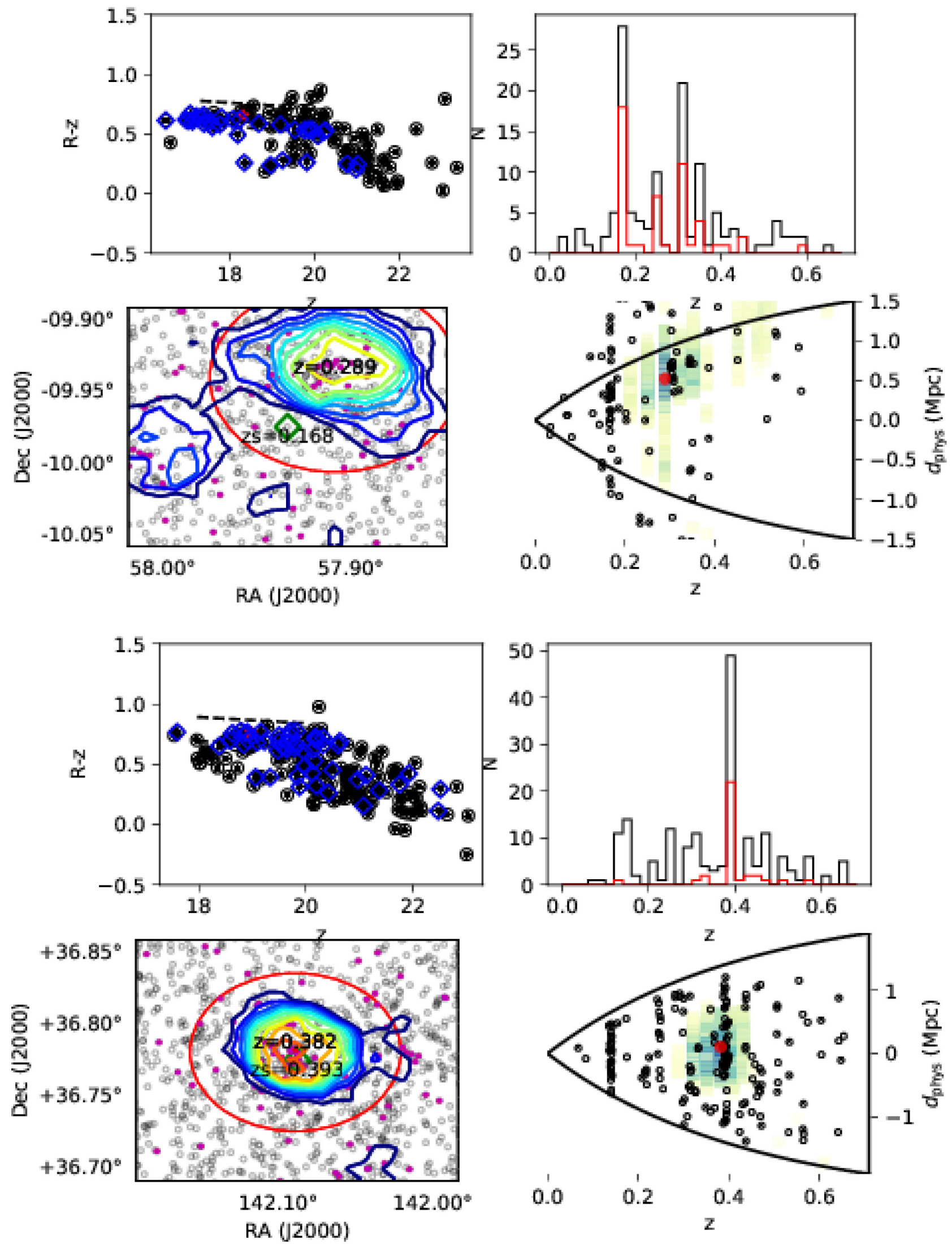

Figure 2. As for previous figure, but fields with multiple spectroscopic overdensities along the line of sight: the zlo_005A,B pair - upper four panels; and zlo_009,10 - lower four panels. See text for details. 

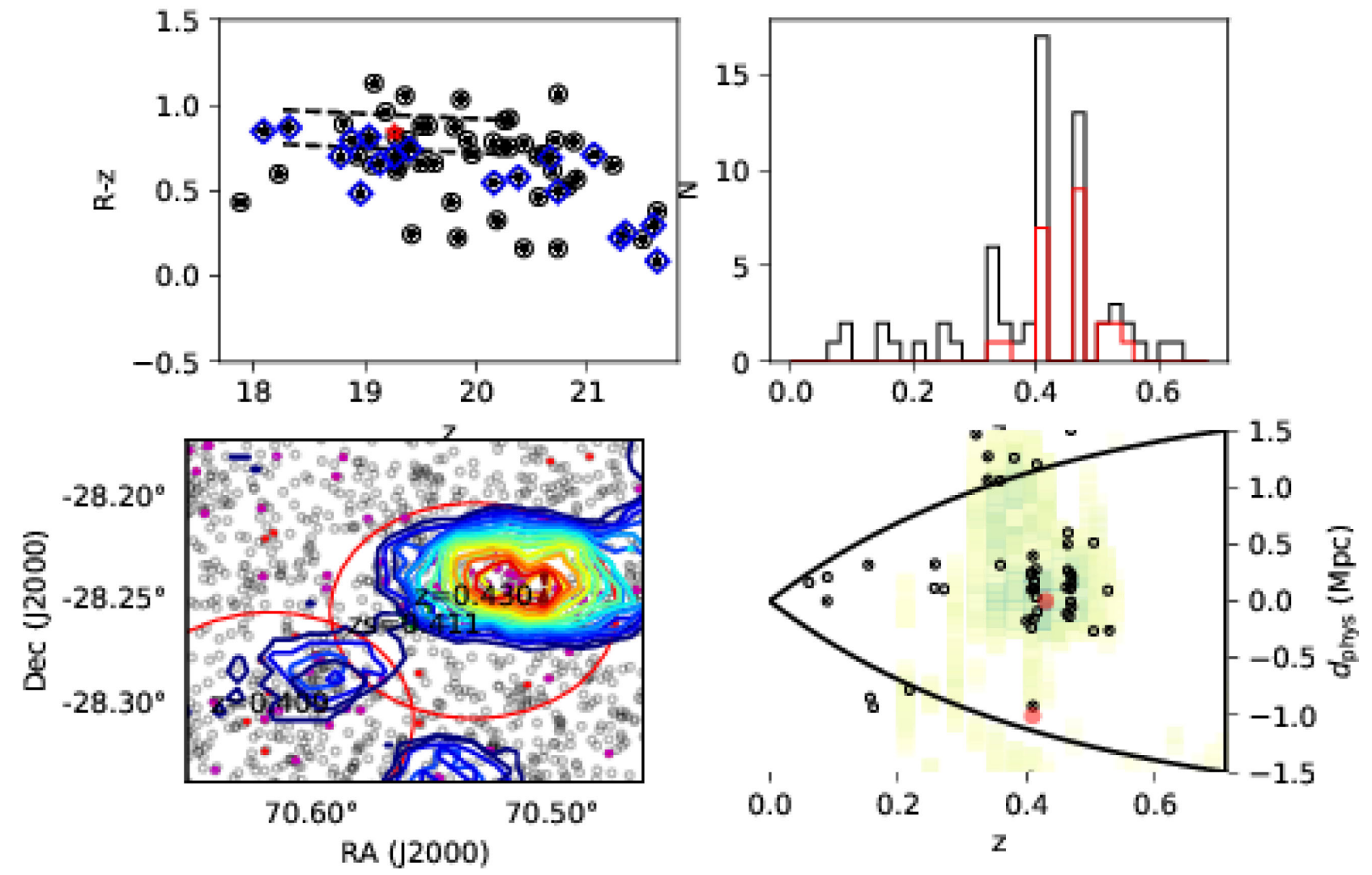

Figure 2 - continued. As previous figure for zlo_006A,B (upper four panels). The two spectroscopic redshift peaks are classed as one system in the photometric cluster catalogue, but could potentially be distinguished by a different deblending algorithm, since the significance map (lower right-hand panel) dips between the two systems. See text for discussion.

cluster candidates. Since the spectroscopic has only produced a handful of successful redshifts, we consider that there is insufficient spectroscopic data in this field to yield an unambiguous result and reject this from further analysis. z1_022 and z1_023 are two peaks in the spectroscopic- $z$ histogram, but the overwhelming majority of red-sequence candidate galaxies are associated with z1_022 peak. Indeed, the z1_023 peak is not spatially concentrated so z1_022 is the correct association with the cluster in the RCS catalogue.

z1_003 shows a modest overdensity at the expected spectroscopic redshift, with about half of the galaxies being appropriate colours for the red-sequence. There are another couple of galaxies with similar colours at slightly higher redshift, however not more than that might be expected from the typical background counts of galaxies of such colours. Given that the primary overdensity has fewer than five confident spectroscopic redshifts, we class this cluster candidate as being tentatively confirmed.

\subsubsection{Previously published clusters}

Several of these clusters have been published in previous work, by our group and one by an independent work.

z1_005 and z1_006 are two components of a projected cluster, RCS 043938-2904.9, first presented in Barrientos et al. (2004) $\left(z_{\text {spec }}=0.961\right.$ and 0.952 , respectively) discussed in detail in Cain et al. (2008).

z1_019 is the primary component of the RCS2319 supercluster reported in Gilbank et al. (2008) and in the recent improved spec- troscopic study of Faloon et al. (2013). In the rest of the paper, we use the improved velocity dispersion calculated in the latter paper.

z1_002 was initially published in Andreon et al. (2008) using, in part, our confirmation spectroscopy that we use here. As noted above, this cluster falls just below the significance cut-off of the photometric cluster catalogue (with $\sigma_{\mathrm{RCS}}=4.3$ ). This is because it lies in a survey region with unusually poor photometry/photometric calibration and so the significance appears to be artificially reduced due to the abnormally low counts of galaxies above the nominal magnitude limit.

\subsubsection{Summary of the z1 sample}

To summarize: The $z \sim 1$ sample consists of 16 red-sequence selected cluster candidates. Of these, four fall below the nominal $\sigma_{\text {RCS }} \geq 5$ significance cut, and so do not appear in the latest catalogue. Three fields have insufficient spectroscopic members $(<5)$ to confirm cluster candidates (two of these three have appeared good enough for tentative confirmation). Of the remaining clusters, eight are regarded as cleanly confirmed. One cluster is a close projection of two systems, which could not be separated using photometric data alone (z1_005,6), and one other cluster (z1_013) show signs of a nearby poorer system/group (z1_014) at small enough projected distance that its member galaxies could potentially affect red-sequence measurements of the primary cluster.

Thus, the following numbers can be used as estimates of the contamination rate in the full RCS- 1 photometric cluster catalogue at these redshifts: serious projected cluster rate $\approx 10$ per cent $(1 / 8$, or $1 / 11$ including the tentatively confirmed systems). This one case 

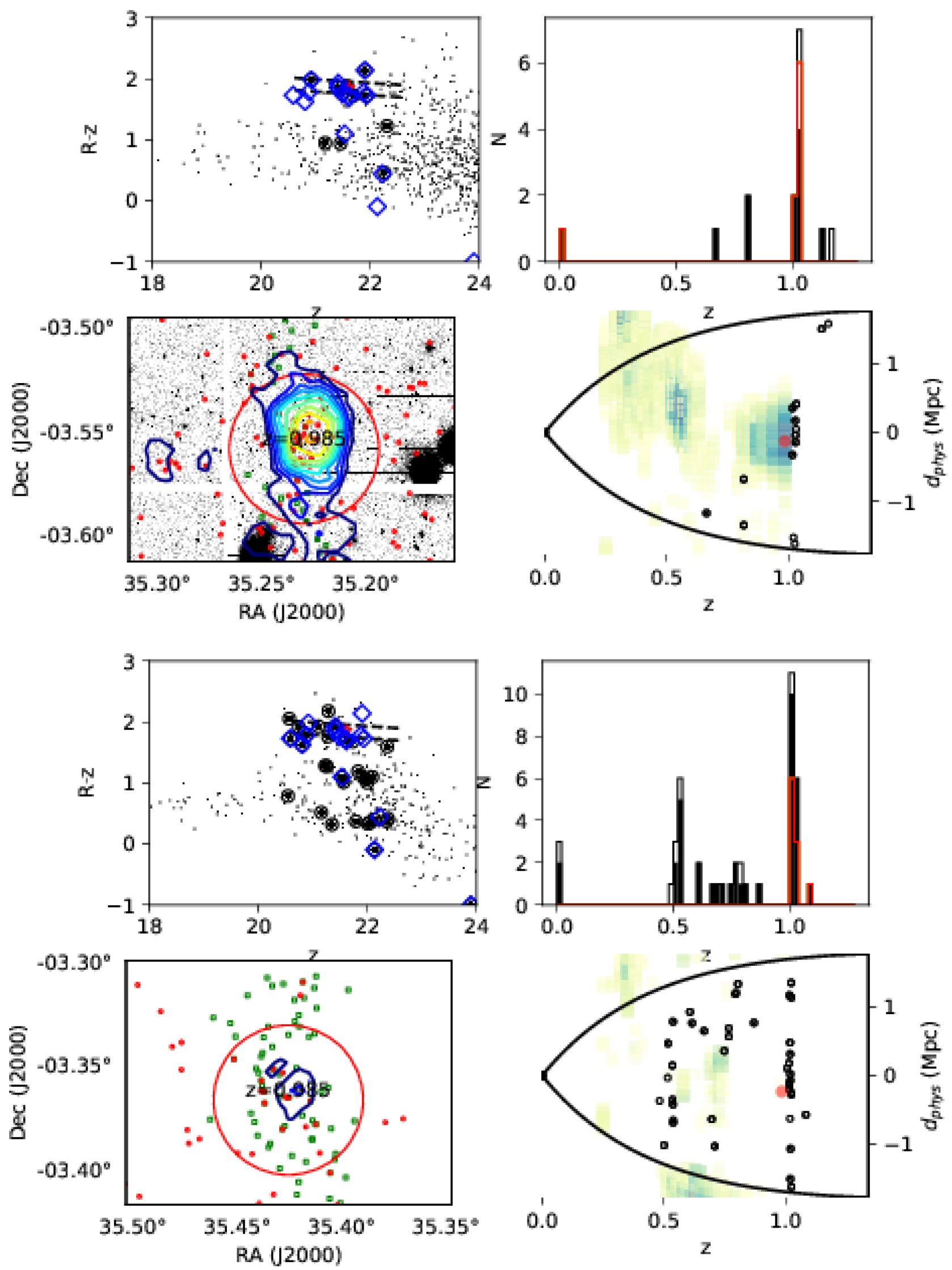

Figure 3. As for preceding figures, but for $z \sim 1$ clusters. Now the two different shadings on the redshift histogram show the two different redshift quality classes: filled denotes classes 1-3; and open is for the lower confidence class 4. See text for more details. z1_001 - top four panels; and z1_002 - bottom four panels. 

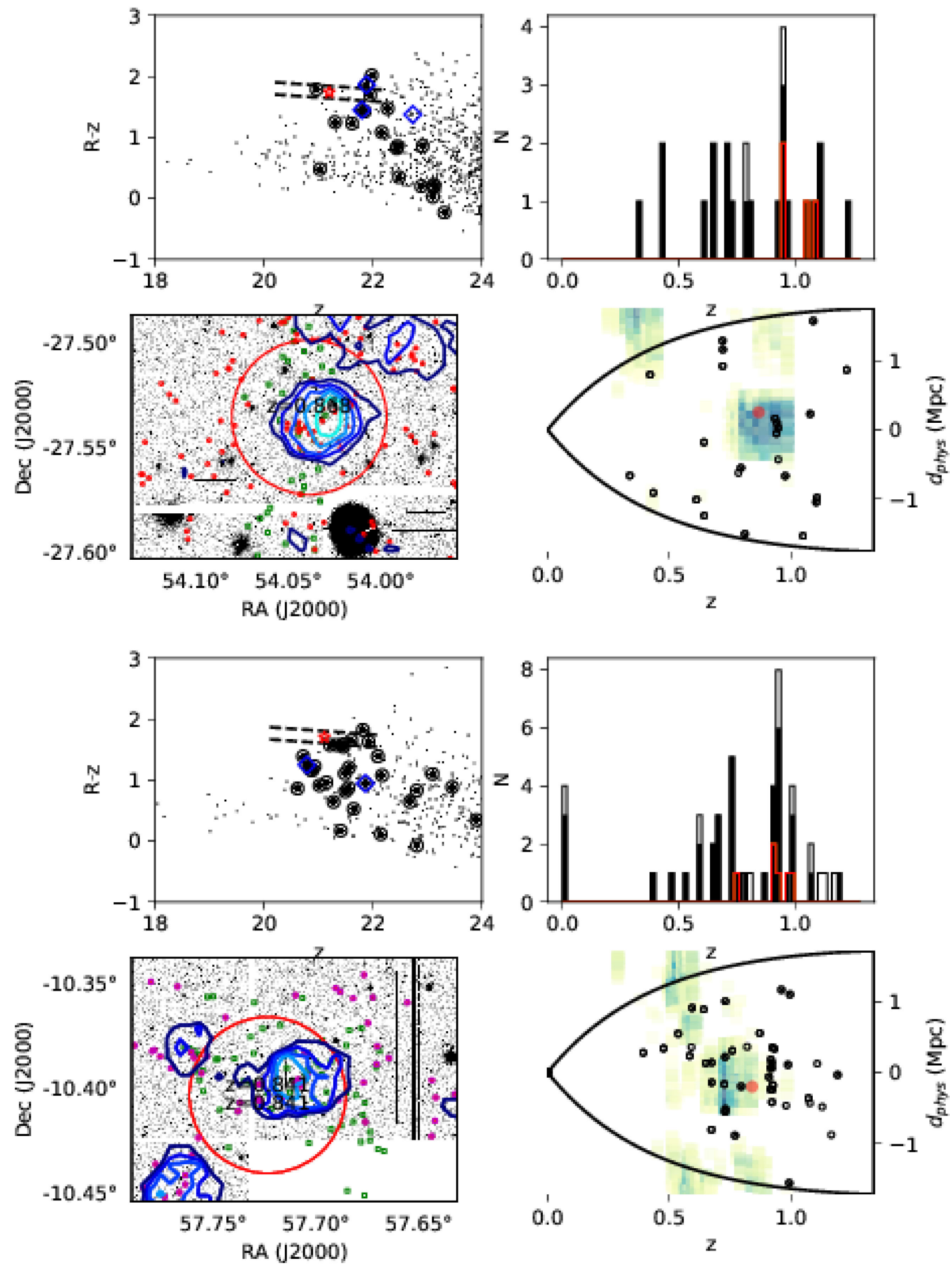

Figure 3 - continued (z1_003 - top four panels, z1_004 - bottom four panels). 

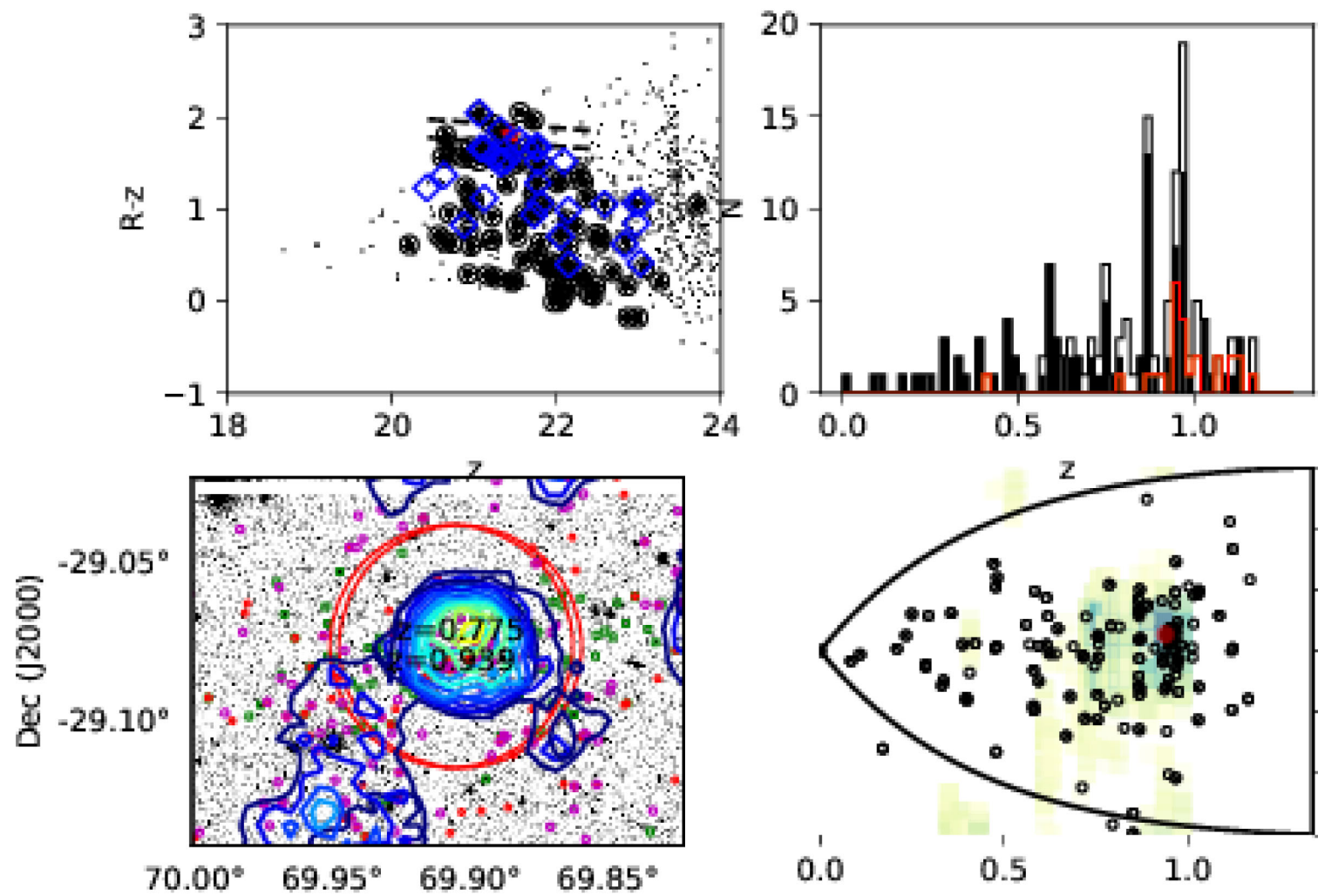

RA (2000)
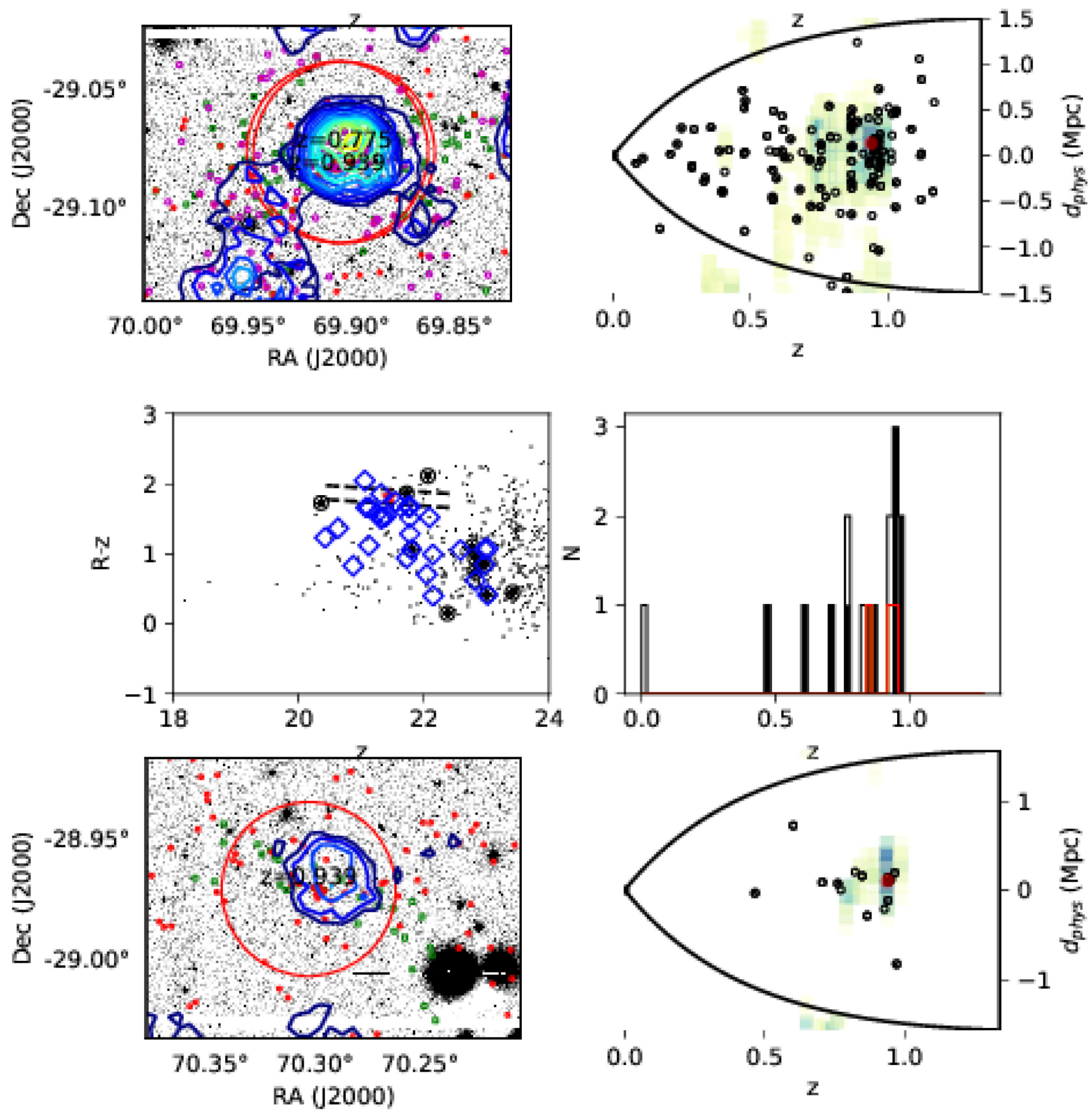

Figure 3 - continued (z1_005,6 - top four panels; and z1_007 - bottom four panels). 

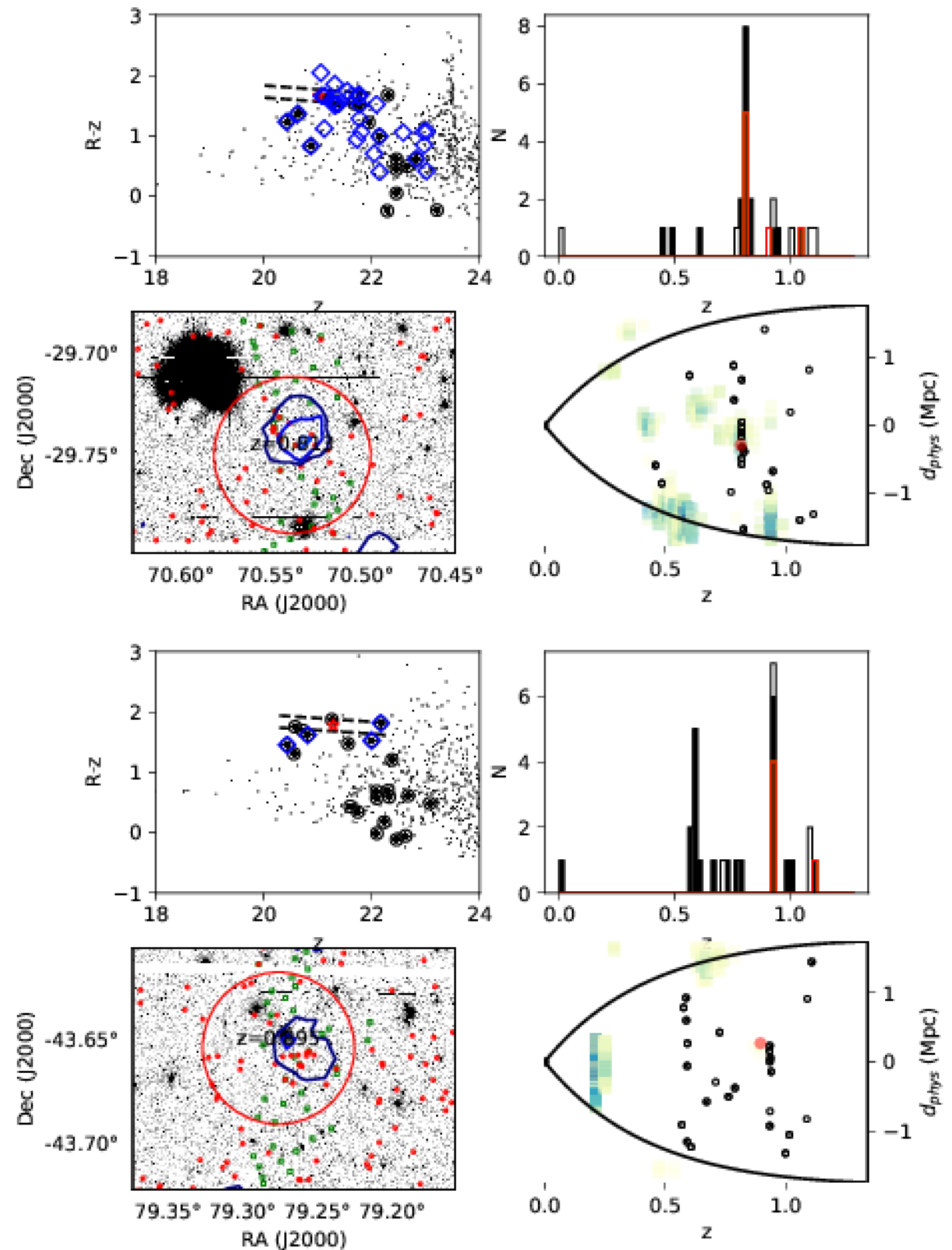

Figure 3 - continued (z1_08 - top four panels; and z1_009 - bottom four panels). 

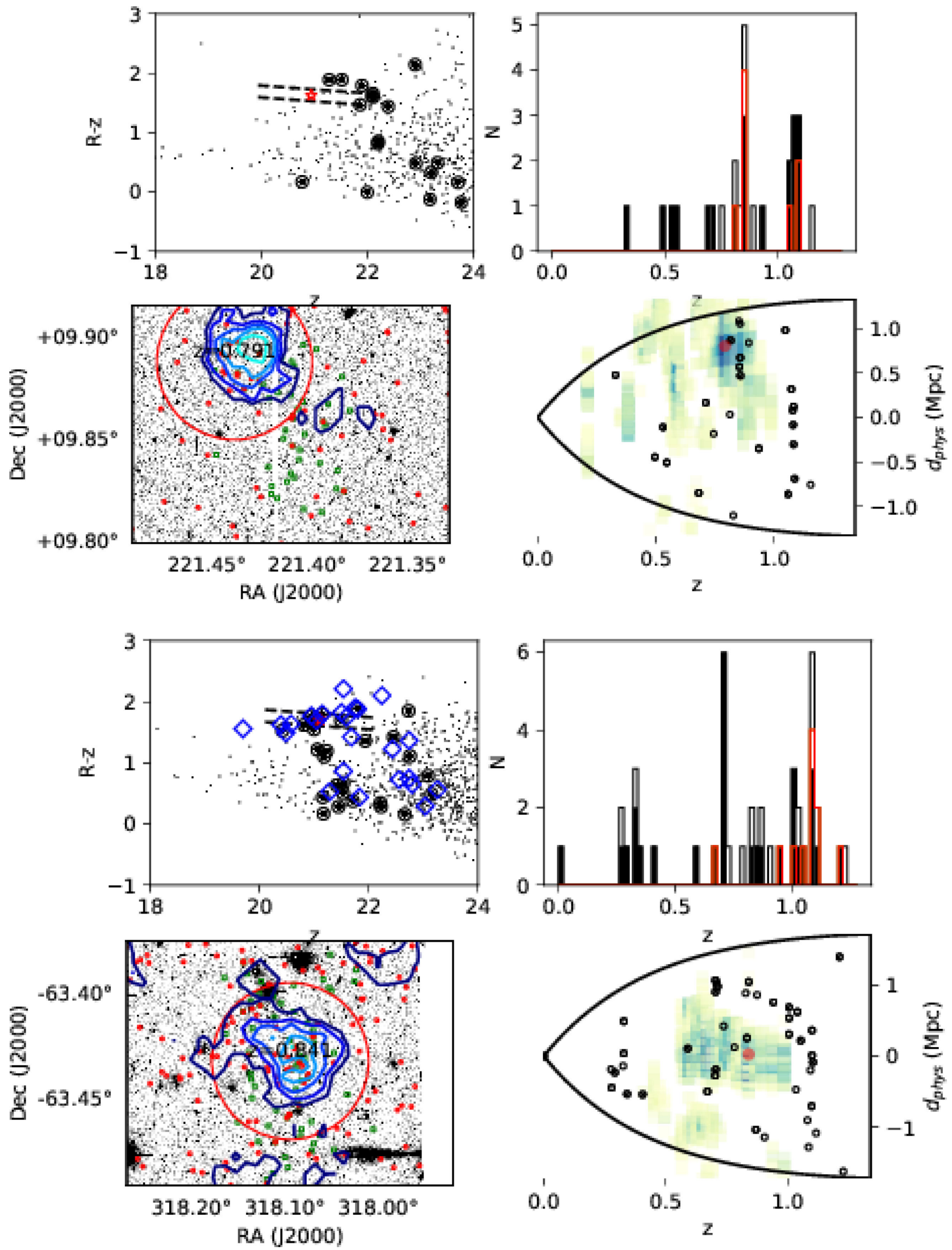

Figure 3 - continued (z1_011,12 - top four panels; and z1_013,14 - bottom four panels). 

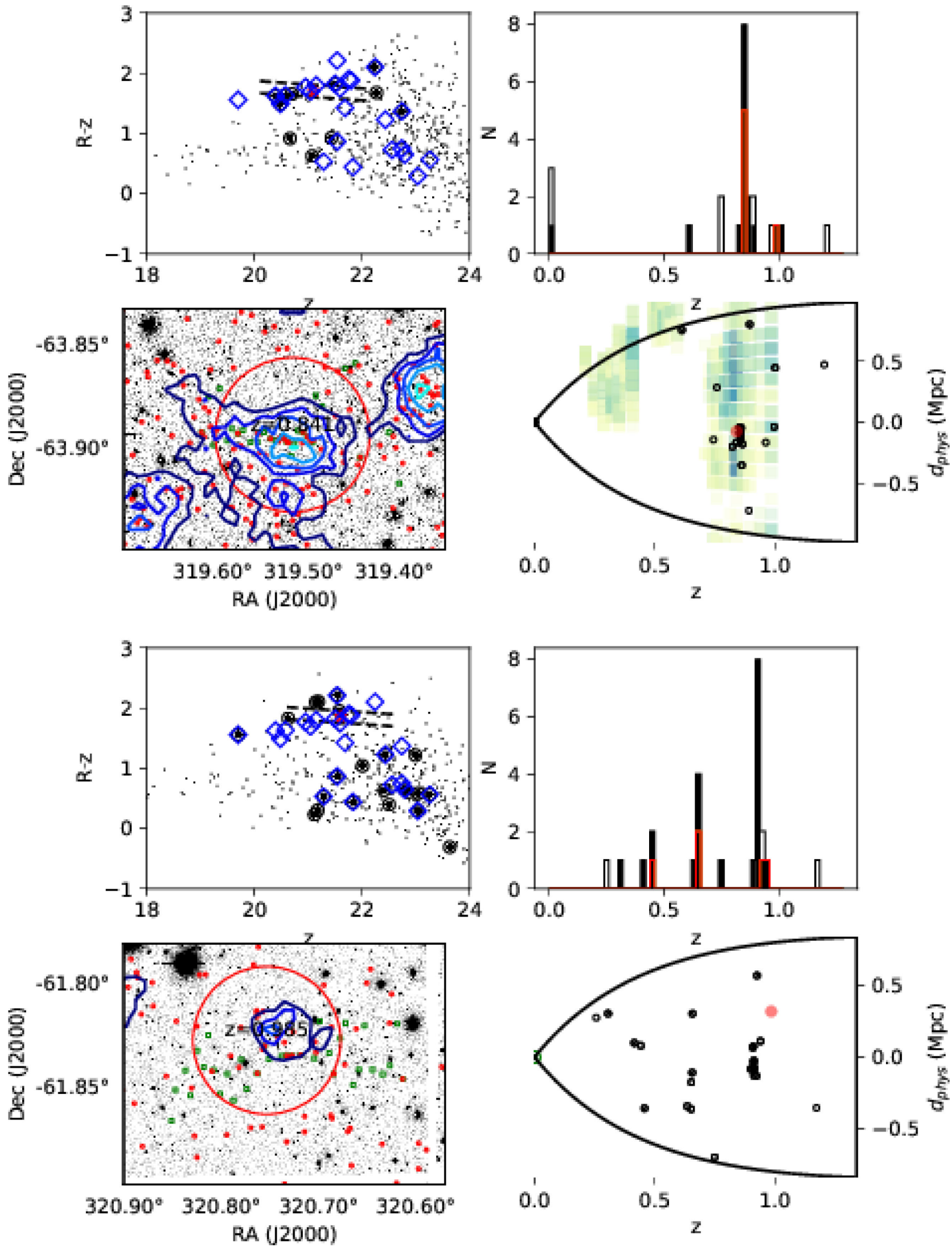

Figure 3 - continued (z1_015 - top four panels; and z1_016 - bottom four panels). 

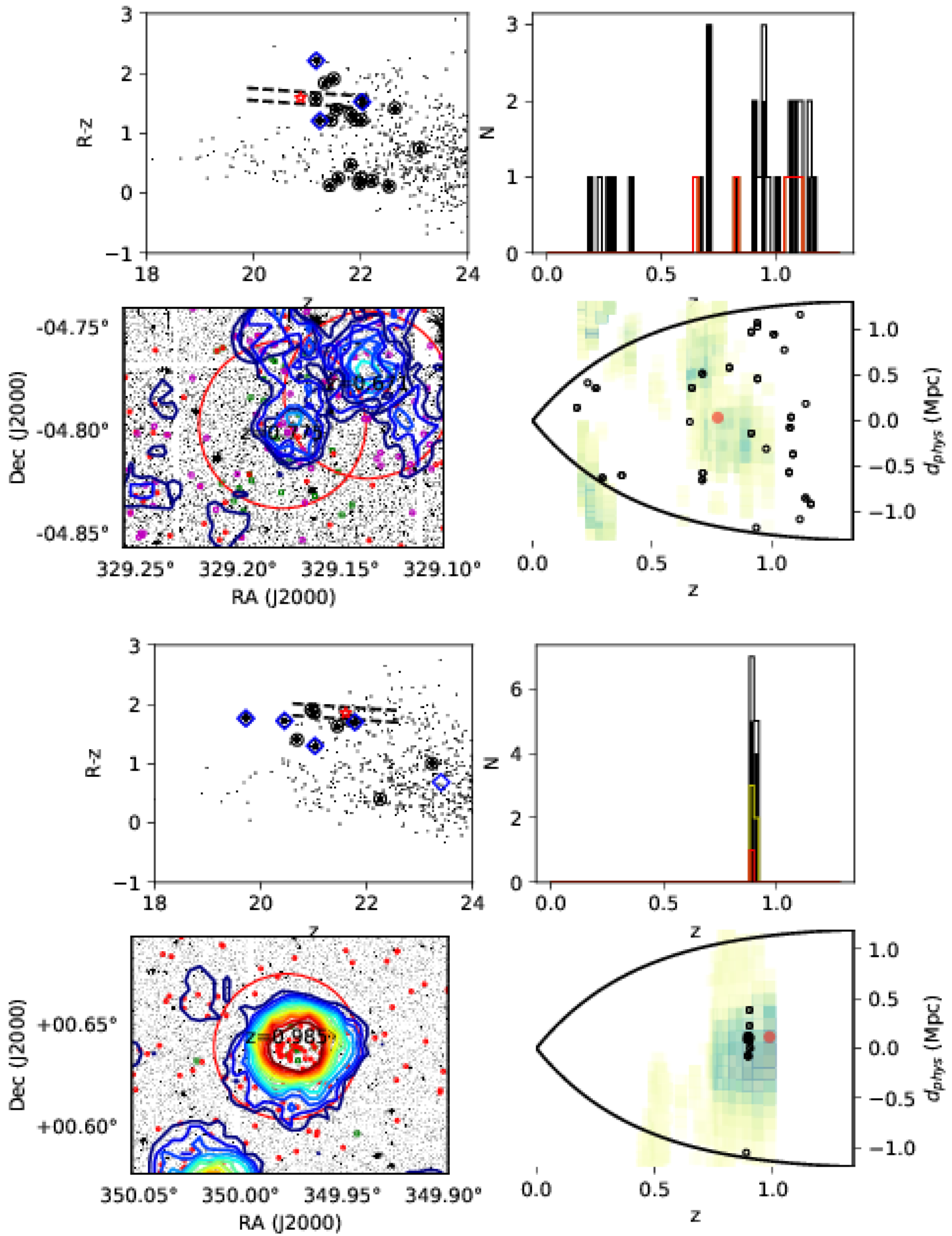

Figure 3 - continued (z1_017,18 - top four panels; and z1_019 - bottom four panels). 

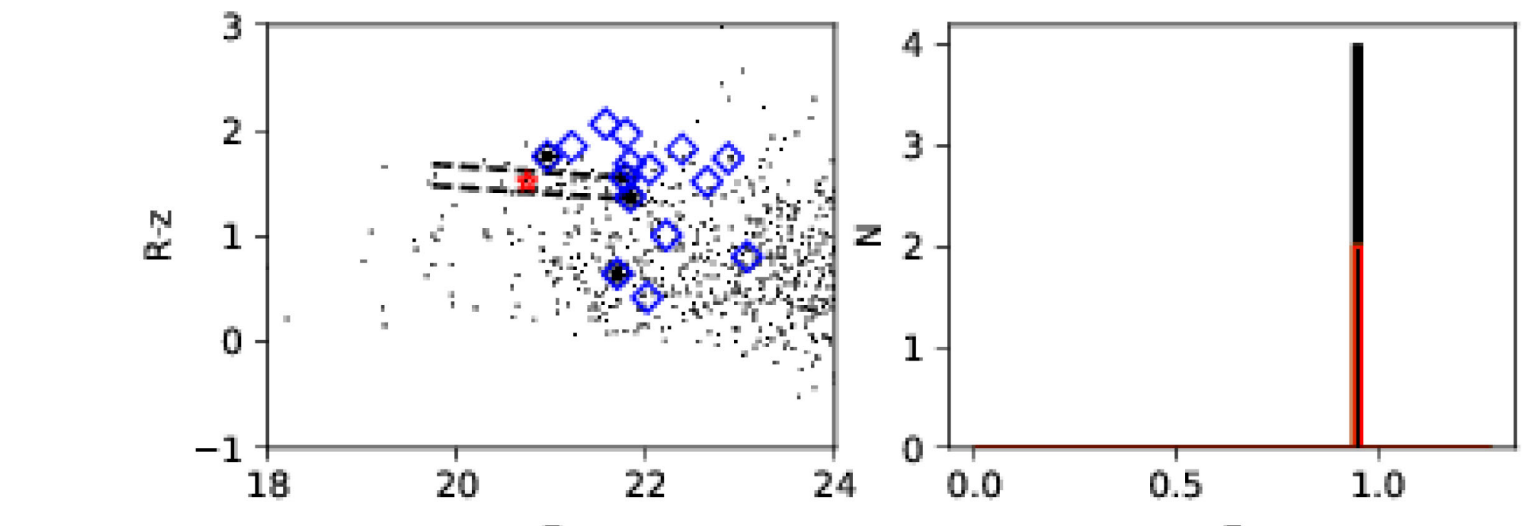

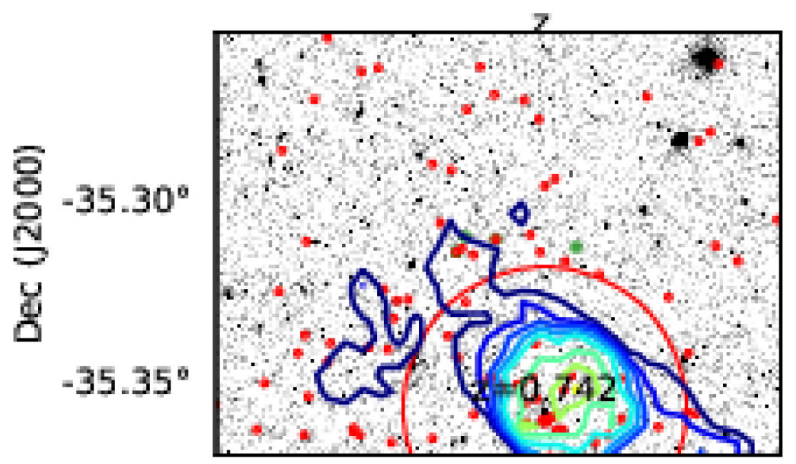

$355.60^{\circ} 355.55^{\circ} 355.50^{\circ} 355.45^{\circ}$

RA (J2000)
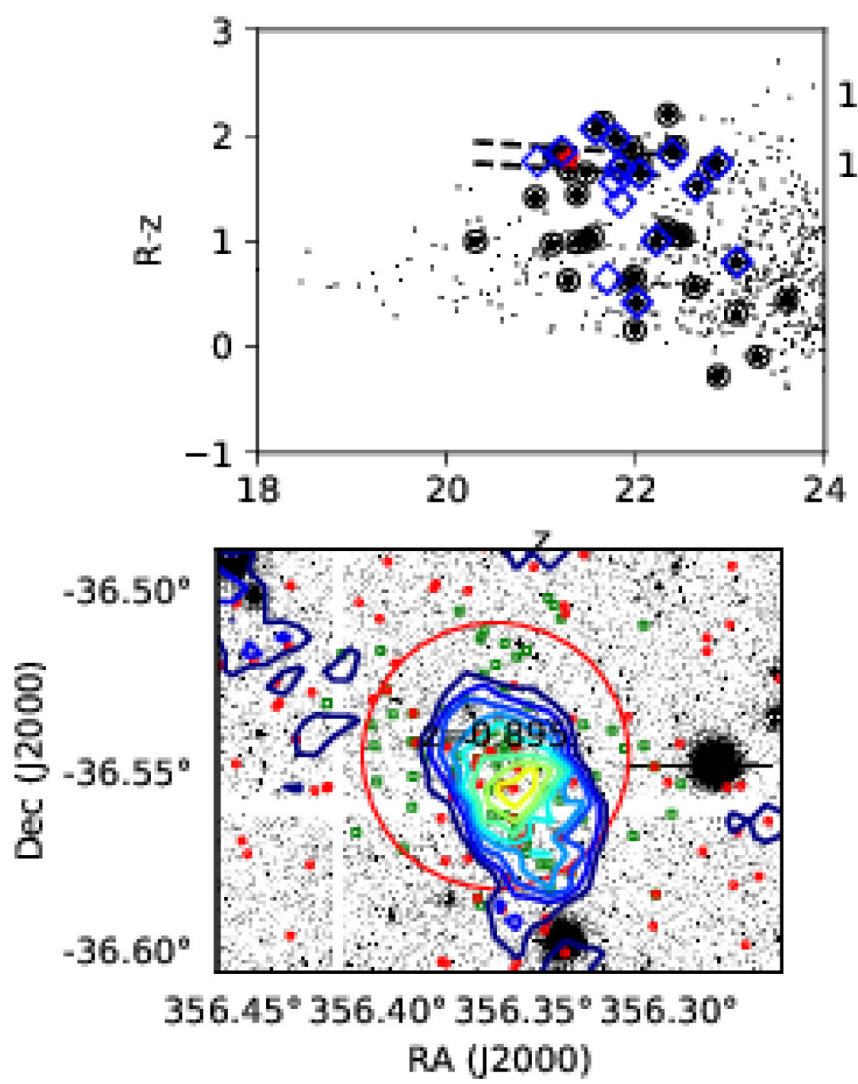

$356.45^{*} 356.40^{\circ} 356.35^{\circ} 356.30^{\circ}$

RA (j2000)
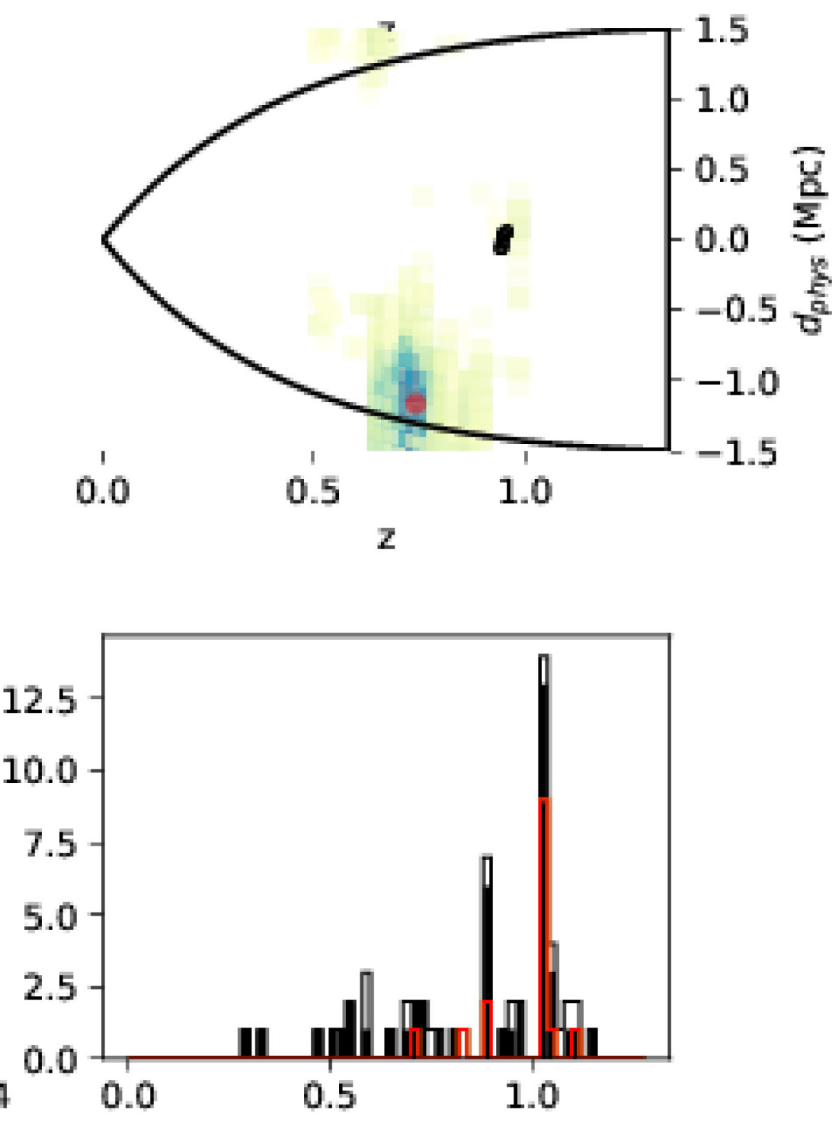

Figure 3 - continued (z1_020 - top four panels; and z1_022,23 - bottom four panels). 
Table 2. Properties of $z \sim 1$ follow-up sample. Columns give: short name, preliminary RCS-1 cluster name (used in other publications), spectroscopic redshift, RA, Dec. of cluster centre in RCS photomoetric cluster catalogue, RCS catalogue photometric redshift, RCS catalogue significance of detection, number of spectroscopic members with quality flag $1-3$, additional comments. “***" in front of the name indicates cluster was not formally identified in full $\left(\sigma_{\mathrm{RCS}} \geq\right.$ 5.0 catalogue) and significance and redshift refer to recovered values. See text for discussion. Velocity dispersion are given where possible (see Section 5.2). Equivalent velocity dispersions from lensing masses, $\sigma_{\text {lens }}$, are taken from Jee et al. (2011).

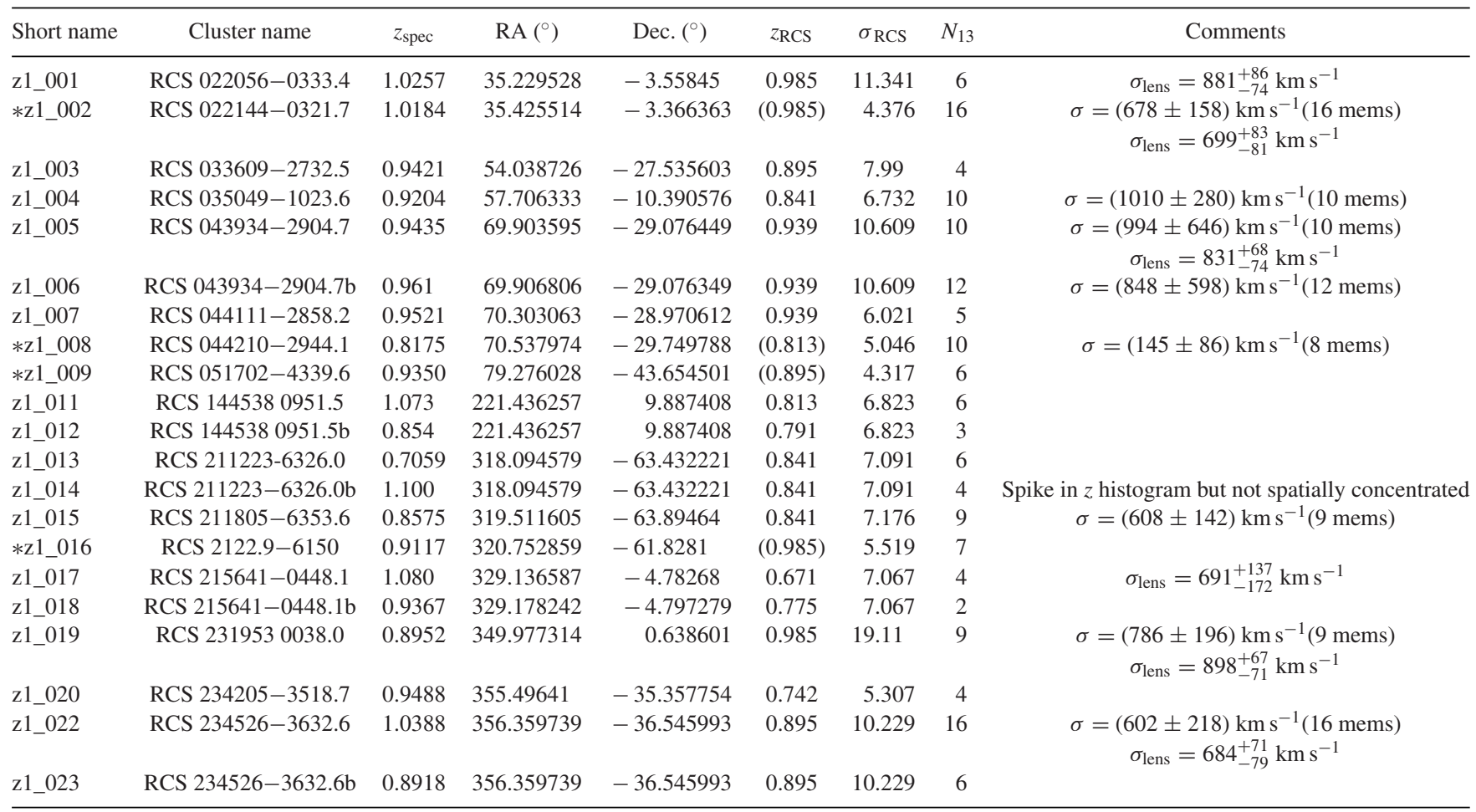

could even be considered to be zero, as a better interpretation is that this is a merging cluster (Cain et al. 2008). Since a further system shows hints of contamination of its red-sequence by a secondary object, the rate of all projections could hence be regarded as $\approx 20$ per cent. This is better thought of as a lower limit, as there may be as yet unseen minor clusters and groups not seen in the current limited spectroscopy which may be contaminating red-sequence selected clusters.

\section{REDSHIFT ACCURACY}

\subsection{SDSS sample}

The results of the mean cluster redshift accuracy above are extended by using data from SDSS DR9 (Ahn et al. 2012). In order to cross-match RCS-1 clusters with SDSS spectroscopic redshifts, the following procedure is used. For each RCS cluster, candidate redsequence galaxies ${ }^{10}$ from RCS photometry are selected. These individual galaxies are cross-correlated with the SDSS spectroscopic catalogue using a matching radius of 2 arcsec. When a match is found, this spectroscopic redshift is assigned to the cluster. No restriction on object type is placed on the SDSS catalogue, so stars and quasars are included as well as galaxies. For each cluster, if more than one of its candidate red-sequence members is assigned a spectroscopic redshift, then the cluster is tagged with the median of these redshifts, but the full range of redshifts assigned to potential

\footnotetext{
${ }^{10}$ Defined as galaxies having a probability of $>10$ per cent of belonging to the model red-sequence slice (Lu et al. 2009)
}

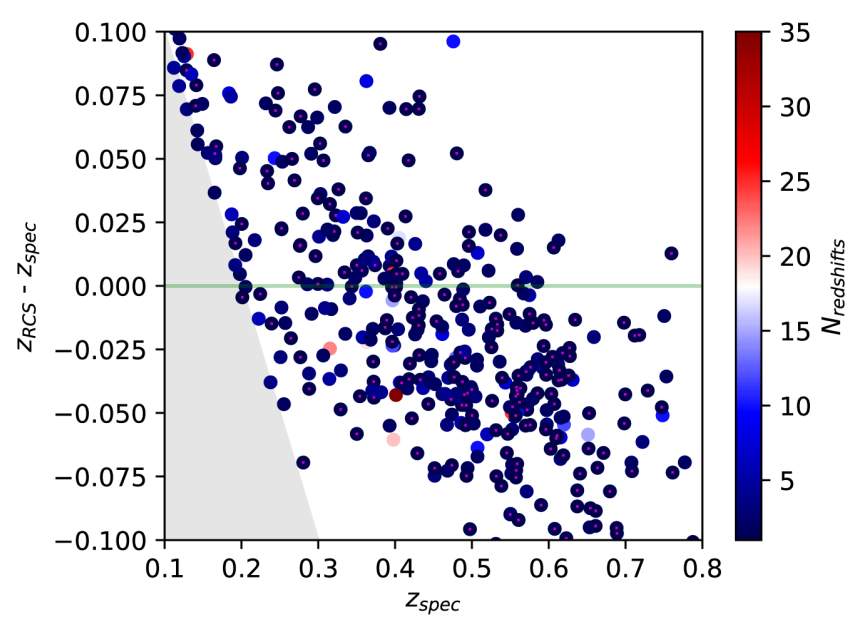

Figure 4. Comparison of SDSS/BOSS spectroscopic redshifts with RCS1 photometric redshifts. Symbol shading is proportional to the number of redshifts matched to candidate cluster members. For clusters with more than one spectroscopic match, the filled circle indicates the median redshift. Data points are plotted randomly offset in $z_{\text {RCS }}$ by \pm 0.015 for clarity.

cluster galaxies is stored. The comparison of these spectroscopic redshifts with the cluster photometric redshifts is shown in Fig. 4.

The agreement is generally very good. There appears to be a tendency for the photometric redshift to be slightly overestimated at the low redshift end $(z \lesssim 0.3)$ and slightly underestimated at the high redshift end. The scatter is generally very small. For systems with multiple matches (larger symbols), the median redshifts all lie close to the one-to-one line. The offset and scatter will be quantified 


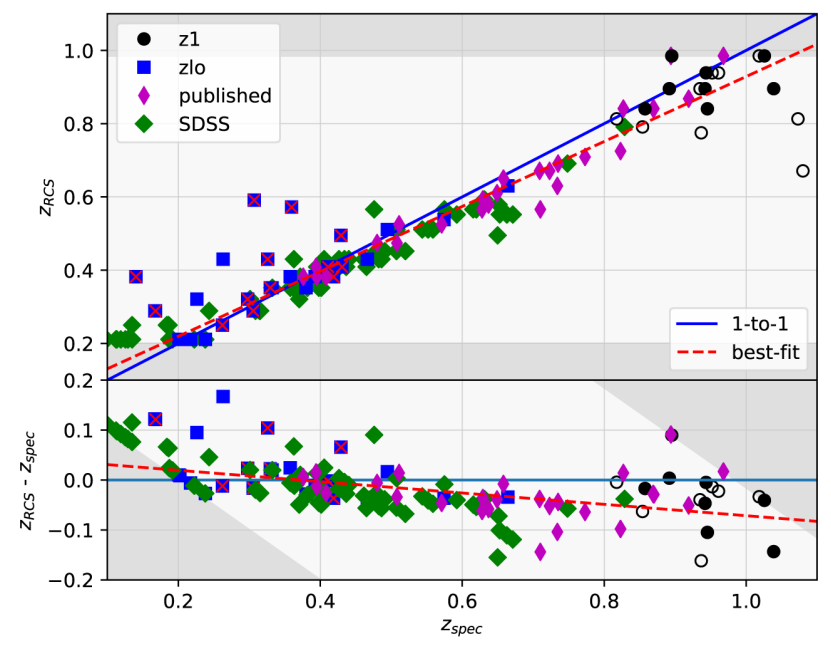

Figure 5. Comparison of RCS cluster-finder redshifts with spectroscopic redshifts. The zlo and z1 samples of this paper are shown as blue squares and black circles, respectively; the SDSS-BOSS data presented in the previous figure are shown as green diamonds (for clusters with more than five spectroscopic matches; and previously published RCS-1 redshifts (from Gilbank et al. 2007; Hicks et al. 2007, 2008; Faloon et al. 2013; Webb et al. 2013) are shown as thin magenta diamonds. The one-to-one line is shown by the solid blue line, and the best fit is shown as the red dashed line. There is a tendency for $z_{\mathrm{RCS}}$ to slightly underestimate $z_{\text {spec }}$. The grey shaded regions indicate regions of exclusion due to the min and max photometric redshift $(0.20,0.985)$ used by the cluster finding algorithm. The lower plot shows the residuals to the one-to-one expectation.

in the next section, using all the best available data. The error bars spanning the 100 per cent range of the matched spectroscopic redshift tend to draw the eye to the worst outliers, making the comparison at first appear exaggerated. This simply means that there are a few extreme outliers/mismatches, generally stars (at the low redshift end) and quasars (at the high redshift end). Out of 1249 spectroscopic matches between RCS red-sequence candidate galaxies and SDSS spectroscopy, 21 are spectroscopically classified as stars (and 14 as QSOs), instead of galaxies. This is, in fact, a very low misclassification rate for point sources in RCS-1 photometry.

\subsection{Combined results}

The result of combining all these RCS-1 follow-up samples (Section 3 plus those with previously published spectroscopic redshifts: Gilbank et al. 2007; Hicks et al. 2007, 2008; Faloon et al. 2013; Webb et al. 2013) with the SDSS LRG redshifts is shown in Fig. 5. Only SDSS LRGs with more than five red-sequence matches per cluster candidate are included. The average relation follows very closely the one-to-one expectation but with a small bias given by $z_{\text {spec }}=1.124 z_{\mathrm{RCS}}-0.043$. The scatter is calculated using the biweight scale estimator (Beers, Flynn \& Gebhardt 1990). It is also readily apparent that at the lowest redshifts, some of the scatter is driven by the hard limit imposed by the $z_{\mathrm{RCS}}>0.20$ photometric redshift limit, the point at which the $(R-z)$ colours become degenerate. The same is true (to a lesser extent, but the spectroscopy is sparser) at the high redshift end, where the limit $z_{\mathrm{RCS}}<0.985$ is used. These excluded regions are indicated in the figures, and a conservative limit of $z_{\mathrm{RCS}}>0.30$ is used when fitting the scatter in the relation. The scatter is $\sigma_{z}=0.047$ and $\sigma_{z}=0.062$ in the redshift ranges $0.3<z_{\mathrm{RCS}}<0.6$ and $0.80<z_{\mathrm{RCS}}<0.985$ (where the data are well sampled), respectively.

\section{DYNAMICAL MASSES AND THE MASS-RICHNESS RELATION}

We now proceed to present a preliminary study of the mass-richness calibration using velocity dispersions to probe the dynamical masses of the clusters.

\subsection{Velocity dispersion estimates}

For the lower redshift sample, where the number of cluster members is generally high, each cluster velocity dispersion was calculated using the shifting gapper technique (Fadda et al. 1996). For the more sparsely populated, higher redshift sample, velocity dispersions were estimated by two of us (EE and DG) in an iterative procedure. Plots of the spatial and velocity information were examined, similar to those shown in Figs 1-3 but with the addition of zoomed-in views around the cluster position and redshift. Starting at the median redshift from a broad cut $\left(\Delta v<15000 \mathrm{~km} \mathrm{~s}^{-1}\right)$ around the nominal cluster centre, galaxies were rejected and the centre recomputed and plots updated. The most useful plots in this case were those showing velocity difference versus projected radius, which were used to identify candidate interlopers via visual estimates of the caustics (Diaferio 1999). In this way, it was possible to identify the special cases, such as projected systems, described above. The cut-off radius used was tuned in a similar way, attempting to include as much data as possible, typically with values $\approx 1 \mathrm{Mpc}$. If neighbouring overdensities threatened to contaminate the measurement, then the radius was manually shrunk to exclude these. This method was found to agree very well with the gapper method on the systems with better statistics. In this way, we were able to carefully flag such problem cases, and obtain an estimate of how sensitive the final velocity dispersions were to the inclusion/exclusion of specific galaxies, choice of centre, etc. To calculate a formal uncertainty on the velocity dispersion, a jackknife method was used.

Objects z1_005 and z1_006 merit some additional comment on the unusually large size of their velocity dispersion uncertainties. As noted in Section 3.2.2, these are two components of a projected system with a small $\left(\sim 4000 \mathrm{~km} \mathrm{~s}^{-1}\right)$ offset in systemic velocities and so deblending their components cleanly to measure two separate velocity dispersions is complicated (see fig. 4 of Cain et al. 2008).

Table 2 shows the comparison between the dynamical measurements and the weak lensing velocity dispersions estimated by Jee et al. (2011) for six $z \sim 1$ clusters from HST data. The excellent agreement shows that the velocity dispersions of the $z \sim 1$ clusters based on relatively few cluster members yield reasonable results, albeit with large uncertainties. Velocity dispersions are converted to masses following Carlberg et al. (1996), i.e. $M_{200}=\frac{3 \sqrt{(3) \sigma^{3}}}{H(z) G}$.

\subsection{Dynamical mass-richness relation}

Richness estimates, $N_{\text {red }}$ and $N_{200}$, and BCG positions, as described in Section 2.1.1, are given in Table 3. To recap, $N_{\text {red }}$ is the background-subtracted count of the numbers of galaxies with colours compatible with the red-sequence $(P>0.1)$ within a $1.0 \mathrm{Mpc}$ radius and brighter than $M^{\star}+1$, centred on the visually identified BCG position. $N_{200}$ is a richness estimated in a scaled radius based on the initial richness measurement, following the method described in van Uitert et al. (2016). These measurements from this work plus additional previously measured RCS-1 clusters with velocity dispersions from optical spectroscopy (Gilbank et al. 2007; Hicks et al. 2007, 2008; Faloon et al. 2013; Webb et al. 2013) are plotted in Fig. 6. 
Table 3. Properties of clusters (z1 and zlo samples) with velocity dispersion estimates available. Columns are: RA, Dec of visually-identified BCG, richness $\left(N_{\text {red }}\right.$ within $1 \mathrm{Mpc}$ radius and counting down to $\left.M^{\star}+1\right)$ and its error, velocity dispersion and its error, short name, mean spectroscopic cluster redshift, number of members used in velocity dispersion estimate.

\begin{tabular}{|c|c|c|c|c|c|c|c|c|c|}
\hline $\begin{array}{l}\mathrm{RA}_{\mathrm{BCG}} \\
\left(^{\circ}\right)\end{array}$ & $\begin{array}{c}\text { Dec.BCG } \\
\left({ }^{\circ}\right)\end{array}$ & $z_{\mathrm{RCS}}$ & $N_{\text {red }}$ & $\delta N_{\text {red }}$ & $\begin{array}{c}\sigma_{\mathrm{vel}} \\
\left(\mathrm{km} \mathrm{s}^{-1}\right)\end{array}$ & $\begin{array}{c}\delta \sigma_{\mathrm{vel}} \\
\left(\mathrm{km} \mathrm{s}^{-1}\right)\end{array}$ & Short name & $z_{s}$ & $N_{\text {mem }}$ \\
\hline 35.874200 & 1.315530 & 0.430 & 12.0 & 3.9 & 386 & 72 & zlo_001 & 0.440 & 21 \\
\hline 36.007400 & -2.461610 & 0.382 & 22.5 & 5.1 & 601 & 82 & zlo_003 & 0.358 & 36 \\
\hline 53.562300 & -28.410900 & 0.630 & 29.9 & 5.7 & 468 & 75 & zlo_004 & 0.664 & 73 \\
\hline 70.515573 & -28.246654 & 0.430 & 21.2 & 4.9 & 684 & 108 & zlo_006B & 0.466 & 12 \\
\hline 78.902500 & -43.426400 & 0.409 & 24.7 & 5.5 & 653 & 194 & zlo_007 & 0.424 & 15 \\
\hline 142.088000 & 36.774600 & 0.382 & 24.0 & 5.3 & 757 & 69 & zlo_010s & 0.140 & 50 \\
\hline 142.546000 & 38.691200 & 0.409 & 13.9 & 4.2 & 567 & 82 & zlo_011 & 0.431 & 24 \\
\hline 170.214870 & 25.462746 & 0.250 & 9.9 & 3.5 & 327 & 104 & zlo_012s & 0.307 & 16 \\
\hline 201.355000 & 29.342800 & 0.382 & 8.5 & 3.4 & 327 & 75 & zlo_015Bs & 0.290 & 18 \\
\hline 221.655000 & 9.011840 & 0.525 & 4.4 & 2.8 & 696 & 136 & zlo_016 & 0.238 & 30 \\
\hline 221.781000 & 9.821440 & 0.211 & 31.5 & 5.9 & 739 & 58 & zlo_017 & 0.202 & 97 \\
\hline 349.627000 & -0.411378 & 0.352 & 18.5 & 4.8 & 572 & 88 & zlo_027 & 0.380 & 27 \\
\hline 57.708323 & -10.397490 & 0.841 & 6.9 & 3.3 & 1010 & 280 & z1_004 & 0.920 & 10 \\
\hline 69.909335 & -29.081595 & 0.939 & 15.5 & 4.3 & 1080 & 320 & z1_006 & 0.961 & 12 \\
\hline
\end{tabular}

The fitting of the mass-richness relation follows a similar method to that outlined in Andreon et al. (2008) using a Bayesian analysis (implemented in pymc $2^{11}$ ), modelling the relation as a power law of the form $M_{200}=A N^{\alpha}$, with a Gaussian intrinsic scatter (in $\log (M), \log (N))$, with uncertainties in all measurements including our estimates of the measurement uncertainties (see their appendix B for a concise overview of the approach).

In a study of this kind, the limiting factor will always be the modest number of galaxies used to estimate velocity dispersions in the least populated systems. We reiterate that we have used a manual, iterative technique to make the cleanest possible sample with the available data: tuning cut-off radii and velocity cuts; removing neighbouring structures; examining the choice of BCG used for cluster centring, etc. Where a range of plausible options exist, these always gave results comparable to the random uncertainties estimated from the jackknife velocity dispersions. Additional systematics (such as the luminosity of the galaxies used, e.g. Old, Gray \& Pearce 2013) will also give uncertainties comparable to, or smaller than, the (broad) random uncertainties.

A detailed examination of the mass-richness relation derived from these and additional spectroscopic data, including the substantial IMACS sample, will be presented in a forthcoming paper. For this work, we are most concerned with studying the general trend and potential systematics caused by the red-sequence selection method. To this end, a local sample measured in exactly the same way as for the RCS-1 clusters is shown for clusters from the WIde-field Nearby Galaxy-cluster Survey (WINGS, Fasano et al. 2006) at $z \sim 0.05$. The construction of this sample is described in Ellingson et al. (in preparation). The RCS-1 clusters follow the same general relation as the WINGS clusters, albeit with larger scatter. In order to examine whether part of the scatter may be driven by an evolving $N_{\text {red }}-\sigma$ relation, the RCS- 1 data points are coded ac-

\footnotetext{
${ }^{11}$ https://github.com/pymc-devs/pymc
}

cording to the two redshift bins (zlo and $\mathrm{z} 1$ ). It is interesting to note that the $z \sim 1$ outliers all tend to lie above the relation, having a higher velocity dispersion at a given richness. For the best-fitting relations to our data plotted in Fig. 6, only the zlo points are considered $(z<0.7)$. A Bayesian linear regression is used, allowing for intrinsic scatter and measurement uncertainties on both axes. For a fit of $M_{200}=A N^{\alpha}$, we find $\log A=(14.40 \pm 0.07) \mathrm{M}_{\odot}$ and $\alpha=0.86 \pm 0.08$ (25-75 per cent credible intervals).

A similar trend was recently seen by van Uitert et al. (2016) using RCS-2 data and weak lensing-inferred masses from richnessselected stacks of clusters. They interpreted the evolution seen in the mass-richness relation to be due to the build-up of the redsequence. van Uitert et al. (2016) measured a best-fitting slope, $\alpha$, in the redshift range $0.35<z<0.55$ of $\alpha=0.86 \pm 0.08$, which is consistent with our zlo fit of $\alpha=1.1 \pm 0.45$, and also with the maxBCG result of $\alpha=0.98 \pm 0.07$, but at slightly lower redshift. We note in passing that our normalization is slightly lower than the two above works by about $0.25 \mathrm{dex}$ at $N_{200} \sim 15$. A detailed comparison is beyond the scope of this work. The slope measurements should be less sensitive to possible systematics such as calibration differences in either variable. See van Uitert et al. (2016) for a more complete summary of recent mass-richness relation measurements.

We now consider other red-sequence selection effects that might increase the scatter in this relation.

\subsubsection{Effect of redshift uncertainty on richness}

Although the scatter in the average photometric-spectroscopic redshift relation is small, as shown above. Some of the larger photometric redshift outliers may have a significant impact on the velocity dispersion-richness relation.

The redshift enters the richness estimate, $N_{\text {red }}$, in three ways. First, since a physical aperture is used to count galaxies, the redshift enters via the angular size-distance relation. Overestimating the redshift will thus result in using a smaller angular counting radius and 

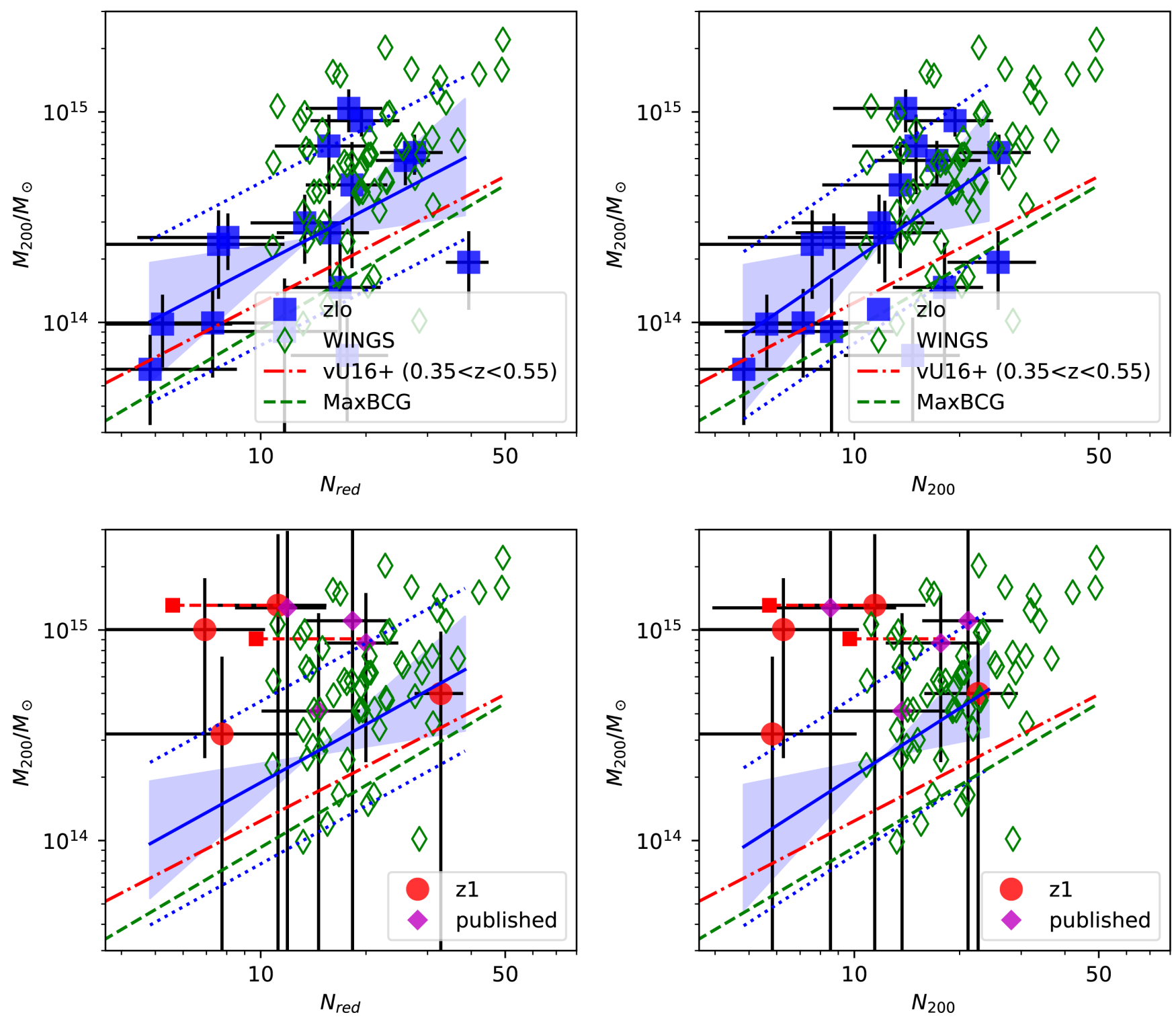

Figure 6. Richness ( $N_{\text {red }}$ left-hand panels, $N_{200}$ right-hand panels)-dynamical mass relations for all RCS-1 clusters with previously published velocity dispersions and those in this paper, data points colour coded by sample. Dashed horizontal lines joining pairs of data points show revised richness estimates for projected systems, by splitting the richness equally between two systems at the same dynamical mass. Also shown are data from the local $(z \sim 0.05)$ WINGS clusters. The RCS-1 clusters follow the same general relation as the WINGS clusters, but with several apparently larger outliers. Lines with shaded regions show the mean and 5 per cent-95 per cent critical intervals for the best-fitting relations to the zlo RCS1 clusters, dotted bounds give the $1 \sigma$ scatter for the same relations, respectively. The higher redshift points ( $\mathrm{z} 1$ and previously published points) all systematically lie towards the higher mass end of the plot. This may suggest that either the richness-mass relation evolves, or the dynamical masses of the higher redshift clusters are more likely to be overestimated due to unrelaxed systems. Also shown are the relations measured by van Uitert et al. (2016) and the MaxBCG results. See text for discussion.

underestimating $N_{\text {red }}$. Secondly, the redshift dictates the counting limit in terms of magnitudes past $M^{\star}$. Overestimating the redshift will result in candidate cluster members being counted to a deeper limit. This overcounting applies not only to the candidate cluster members, but also to the background galaxy estimates. It is not immediately obvious what the net effect of this overcounting/undercounting will be, since it depends on the relative numbers in the cluster aperture to those in the same area in the background. Finally, the redshift dictates the colours of the galaxies chosen for the colour slice. Again, the net difference in the number counts depends on the relative numbers in the cluster versus background apertures in the various colour slices, and so it is not immediately obvious what the effect of overestimating the redshift has on the number counts, with respect to the colour slice used.

We now examine the effect of attempting to correct for small redshift offsets by replacing the photometric redshifts with spectroscopic redshifts and remeasuring $N_{\text {red }}$. There is a subtlety involved in attempting this correction, which involves the third point above. Since the clusters were detected using colour selection (specifically by choosing the colour slice in which the significance of an overdensity is maximized), shifting the colours of galaxies counted to another colour slice (given by the spectroscopic redshift) may systematically reduce the significance/richness. Thus, it matters whether the 'wrong' slice was chosen due to (a) photometric calibra- 
tion error of the survey data, or (b) correct photometric calibration but a neighbouring slice being chosen because the formal significance is almost equally high in more than one slice (and possibly just noise on the significance measurement resulted in a different slice being chosen). In case (a), the size of the counting aperture should be updated to that given by the spectroscopic redshift; the colour slice used should not be updated (as the overdensity is maximized, it is just the absolute calibration of the colour which is incorrect); and whether or not the counting limit is updated would depend on whether the calibration error lies in the $R$-band or the $z$-band, the latter being used for the counting limit. It is not possible to know this final point without further photometric calibration data.

For case (b), all three quantities depending on redshift should be updated to the spectroscopic redshift, and this is the easiest case to test. We replace the photometric redshifts with the colour slice nearest to that given by the spectroscopic redshift and remeasure $N_{\text {red }}$. The vast majority of points are only shifted within the error bars of the richness estimate made using the photometric estimate (not plotted). Indeed, for the few cases where the updated redshift makes a $>1 \sigma$ difference, the points move away from the average relation traced by the WINGS clusters.

Some of the effects of case (a) can be tested. Simply changing the counting radius using the physical size given by the spectroscopic redshifts leads to relatively little change in $N_{\text {red }}$ from the photometric redshift-derived values. As mentioned, it is not possible to tell how the numbers should shift by correcting the photometric zero-point, and so this is the best that can be done with the current data. We can however conclude that correcting all measurements using the spectroscopic redshift of each value (the assumption of case b errors) leads to a significant reduction in the number of obvious outliers in the mass-richness relation for those RCS-1 clusters with velocity dispersions.

\subsubsection{Effects of projection}

As mentioned, several clusters (zlo_005B, zlo_010s, z1_006) show line-of-sight projections, close enough that their red-sequences overlap and hence their richness measurements cross-contaminate each other. Ideally, one would like to be able to deblend the effects of this projection, splitting the richnesses according to the relative contribution of each cluster. This may be possible if mass estimates are available for each component and the scaling between mass and richness is known a priori. In the absence of this information, the next best option is to simply split the measured $N_{\text {red }}$ value equally between each component ${ }^{12}$. For the clusters showing signs of projection and appearing in the mass-richness plots, these are now replotted in Fig. 6, applying this correction as described. In all three cases, splitting the richness equally in half between two components moves the clusters further from the mass-richness relation. In the case of z1_006, this merging cluster is almost certainly not in virial equilibrium and so the velocity dispersion does not reliably trace the dynamical mass of the cluster. Indeed, this system already lies further from the average relation than the other two clusters. In the other two cases, the clusters initially lie very close to the mean relation and so halving their richnesses moves them away from it. One explanation of this would be that the initial red-sequence richness is already a reasonable estimate of the true richness, and, thus, contamination by the secondary components is minimal.

\footnotetext{
${ }^{12}$ Or in the case that the second component is clearly much poorer or less massive the first, simply neglect the richness of the second entirely.
}

\section{SUMMARY}

We have presented a comprehensive overview of the accuracy of the RCS's cluster selection technique, primarily aimed at the lower and higher redshift range probed by the survey. Using multiobject spectroscopic observation of cluster candidates, we find that the cluster catalogue essentially identifies all rich, significant clusters $\left(\sigma_{\mathrm{RCS}} \geq 5.0\right)$, as no clusters were seen in the spectroscopy, which were missed by the photometric cluster detection. An exception to this is where multiple systems are superposed along the line of sight. Such superpositions should be considered in two categories: those which are physically related systems and those which are much farther apart. In the former category, clusters that are separated by only a few hundred $\mathrm{km} \mathrm{s}^{-1}$ (e.g. RCS043934-2904.7A,B) will never be separable by such a photometric technique. However, it is quite surprising that the incidence of such systems is relatively low in the RCS- 1 sample $(0 / 24$ at $0.2 \leq z \lesssim 0.6$ and $1 / 8$ at $z \sim 1)$ since galaxy-selected cluster methods make no requirements on the dynamical state of the system. The second category depends on the way in which the $3 \mathrm{D}$ significance maps are deblended into separate structures. Clusters along the same sight-line at, say, $z=0.2$ and 0.4 should potentially be resolvable since the redshift slices are well-separated in colour. With further examples of such systems, the deblending parameters for cluster-finding can be more precisely tuned to correctly recover such cases, without overly deblending genuinely single clusters.

(i) The red-sequence photometric cluster redshift in RCS-1 (from $(R-z)$ colour alone) is accurate to around $\delta z=0.05$ at $0.3 \lesssim z \lesssim$ 0.6 and $\delta z=0.07$ at $0.8 \lesssim z \lesssim 1.0$.

(ii) The incidence of serious projection (where both components share a significant fraction of the red-sequence galaxies causing the detection) in this sample is $0 / 24$ at $0.2 \leq z \lesssim 0.6$ and $1 / 8$ at $z \sim 1$, including more minor contamination levels, lower limits to the rates are estimated at $1 / 24$ and $2 / 8$, respectively.

(iii) The RCS- 1 clusters show a dynamical mass-richness relation that seems consistent in slope and normalization with local clusters selected from WINGS, albeit with possibly larger scatter. There does not appear to be an obvious systematic difference in this relation between lower and higher redshift clusters.

Future papers will explore the mass-richness calibration and include the remaining large spectroscopy data set from RCS-1 followup.

\section{ACKNOWLEDGEMENTS}

DGG acknowledges support from the National Research Foundation of South Africa. Support for LFB and TA is provided by the Ministry of Economy, Development, and Tourism's Millennium Science Initiative through grant IC120009, awarded to The Millennium Institute of Astrophysics, MAS. LI and LFB are in part supported by CONICYT-Chile grant Basal-CATA PFB-06/2007. HKCY acknowledge support for this project from a Discovery Grant from the National Science and Engineering Research Council of Canada and grants from the Canada Research Chair Program. MC's research is supported by the Transregional Collaborative Research Centre TRR 33.

This paper includes data gathered with the $6.5-\mathrm{m}$ Magellan Telescopes located at Las Campanas Observatory, Chile. This paper also includes data based on observations obtained at the Canada-FranceHawaii Telescope (CFHT), which is operated by the National Research Council of Canada, the Institut National des Sciences de 
l'Univers of the Centre National de la Recherche Scientifique of France, and the University of Hawaii.

Funding for SDSS-III has been provided by the Alfred P. Sloan Foundation, the Participating Institutions, the National Science Foundation, and the U.S. Department of Energy Office of Science. The SDSS-III web site is http://www.sdss3.org/.

SDSS-III is managed by the Astrophysical Research Consortium for the Participating Institutions of the SDSS-III Collaboration including the University of Arizona, the Brazilian Participation Group, Brookhaven National Laboratory, University of Cambridge, Carnegie Mellon University, University of Florida, the French Participation Group, the German Participation Group, Harvard University, the Instituto de Astrofisica de Canarias, the Michigan State/Notre Dame/JINA Participation Group, Johns Hopkins University, Lawrence Berkeley National Laboratory, Max Planck Institute for Astrophysics, Max Planck Institute for Extraterrestrial Physics, New Mexico State University, New York University, Ohio State University, Pennsylvania State University, University of Portsmouth, Princeton University, the Spanish Participation Group, University of Tokyo, University of Utah, Vanderbilt University, University of Virginia, University of Washington, and Yale University.

\section{REFERENCES}

Abell G. O., 1958, ApJS, 3, 211

Ahn C. P. et al., 2012, ApJS, 203, 21

Andreon S., de Propris R., Puddu E., Giordano L., Quintana H., 2008, MNRAS, 383, 102

Angulo R. E., Springel V., White S. D. M., Jenkins A., Baugh C. M., Frenk C. S., 2012, MNRAS, 426, 2046

Barrientos L. F., Gladders M. D., Yee H. K. C., Infante L., Ellingson E., Hall P. B., Hertling G., 2004, ApJ, 617, L17

Beers T. C., Flynn K., Gebhardt K., 1990, AJ, 100, 32

Bleem L. E. et al., 2015, ApJS, 216, 27

Borgani S., Guzzo L., 2001, Nature, 409, 39

Cain B. et al., 2008, ApJ, 679, 293

Carlberg R. G., Yee H. K. C., Ellingson E., Abraham R., Gravel P., Morris S., Pritchet C. J., 1996, ApJ, 462, 32

Chambers K. C. et al., 2016, preprint (arXiv:1612.05560)

Dawson K. S. et al., 2009, AJ, 138, 1271

Diaferio A., 1999, MNRAS, 309, 610

Donahue M. et al., 2001, ApJ, 552, L93

Eke V. R., Cole S., Frenk C. S., 1996, MNRAS, 282, 263

Fadda D., Girardi M., Giuricin G., Mardirossian F., Mezzetti M., 1996, ApJ, 473,670

Faloon A. J. et al., 2013, ApJ, 768, 104

Fasano G. et al., 2006, A\&A, 445, 805

Flaugher B., 2005, Int. J. Mod. Phys. A, 20, 3121

Gal R. R., de Carvalho R. R., Odewahn S. C., Djorgovski S. G., Margoniner V. E., 2000, AJ, 119, 12

Gilbank D. G., Bower R. G., Castander F. J., Ziegler B. L., 2004, MNRAS, 348, 551

Gilbank D. G., Yee H. K. C., Ellingson E., Gladders M. D., Barrientos L. F., Blindert K., 2007, AJ, 134, 282

Gilbank D. G., Yee H. K. C., Ellingson E., Hicks A. K., Gladders M. D., Barrientos L. F., Keeney B., 2008, ApJ, 677, L89

Gilbank D. G., Gladders M. D., Yee H. K. C., Hsieh B. C., 2011, AJ, 141, 94

Gladders M. D., Yee H. K. C., 2000, AJ, 120, 2148

Gladders M. D., Yee H. K. C., 2005, ApJS, 157, 1
Hasselfield M. et al., 2013, J. Cosmol. Astropart. Phys., 7, 008

Hicks A. K. et al., 2007, ApJ, 671, 1446

Hicks A. K. et al., 2008, ApJ, 680, 1022

Hicks A. K. et al., 2013, MNRAS, 431, 2542

Jee M. J. et al., 2011, ApJ, 737, 59

Kettula K. et al., 2015, MNRAS, 451, 1460

Koester B. P. et al., 2007, ApJ, 660, 239

Kravtsov A. V., Vikhlinin A., Nagai D., 2006, ApJ, 650, 128

Le Fevre O., Crampton D., Felenbok P., Monnet G., 1994, A\&A, 282, 325

LSST Science Collaboration et al., 2009, preprint (arXiv:0912.0201)

Lu T., Gilbank D. G., Balogh M. L., Bognat A., 2009, MNRAS, 399, 1858

Majumdar S., Mohr J. J., 2004, ApJ, 613, 41

Maughan B. J., 2014, MNRAS, 437, 1171

Meyers J. et al., 2012, ApJ, 750, 1

Miller C. J. et al., 2005, AJ, 130, 968

Morokuma T. et al., 2010, PASJ, 62, 19

Old L., Gray M. E., Pearce F. R., 2013, MNRAS, 434, 2606

Planck Collaboration XII, 2011, A\&A, 536, A12

Planck Collaboration XXIX, 2013, A\&A, 571, A29

Pratt G. W., Croston J. H., Arnaud M., Böhringer H., 2009, A\&A, 498, 361

Rozo E., Bartlett J. G., Evrard A. E., Rykoff E. S., 2014, MNRAS, 438, 78

Rykoff E. S. et al., 2014, ApJ, 785, 104

Saro A. et al., 2015, MNRAS, 454, 2305

Sehgal N. et al., 2013, ApJ, 767, 38

Sunyaev R. A., Zeldovich Y. B., 1972, Comments Astrophys. Space Phys., 4,173

van Uitert E., Gilbank D. G., Hoekstra H., Semboloni E., Gladders M. D., Yee H. K. C., 2016, A\&A, 586, A43

Webb T. M. A. et al., 2013, AJ, 146, 84

Yee H. K. C., Ellingson E., Carlberg R. G., 1996, ApJS, 102, 269

Yee H. K. C. et al., 2000, ApJS, 129, 475

Yu H., Tozzi P., Borgani S., Rosati P., Zhu Z.-H., 2011, A\&A, 529, A65

${ }^{1}$ South African Astronomical Observatory, PO Box 9, Observatory, 7935, South Africa and Centre for Space Research, North-West University, Potchefstroom 2520, South Africa

${ }^{2}$ Instituto de Astrofísica, Pontificia Universidad Católica de Chile, Avda. Vicuña Mackenna 4860, Macul, Casilla 306, Santiago 22, Chile

${ }^{3}$ Center for Astrophysics and Space Astronomy, Department of Astrophysical and Planetary Science, UCB-389, University of Colorado, Boulder, CO 80309, USA

${ }^{4}$ Department of Astronomy and Astrophysics, University of Toronto, 60 St. George Street, Toronto, ON M5S 3H8, Canada

${ }^{5}$ Departamento de Ciencias Fisicas, Universidad Andres Bello, Fernandez Concha 700, Las Condes, Santiago, Chile

${ }^{6}$ Department of Astronomy and Astrophysics, University of Chicago, 5640 S. Ellis Ave., Chicago, IL, 60637, USA

${ }^{7}$ Department of Physics and Astronomy, York University, 4700 Keele St., Toronto, ON, M3J 1P3, Canada

${ }^{8}$ Department of Physics and Astronomy, University of Kentucky, 505 Rose Street, Lexington, KY 40506, USA

${ }^{9}$ Zentrum für Astronomie, Institut für Theoretische Astrophysik Philosophenweg 12, D-69120 Heidelberg, Germany

${ }^{10}$ Leiden Observatory, Leiden University, P. O. Box 9513, NL-2300 RA Leiden, The Netherlands

${ }^{11}$ Department of Physics and Astronomy, University of Utah, Salt Lake City, UT 84112, USA

${ }^{12}$ Australian Astronomical Observatory, North Ryde, NSW 2113, Australia

${ }^{13}$ Institute of Astronomy, Graduate School of Science, The University of Tokyo, 2-21-1 Osawa, Mitaka, Tokyo 181-0015, Japan

This paper has been typeset from a $\mathrm{T}_{\mathrm{E}} \mathrm{X} / \mathrm{LT}_{\mathrm{E}} \mathrm{X}$ file prepared by the author. 